\title{
The slingshot effect: a possible new laser-driven high energy acceleration mechanism for electrons
}

\author{
Gaetano Fiore ${ }^{1,3}$, Renato Fedele ${ }^{2,3}$, Umberto de Angelis ${ }^{3}$, \\ ${ }^{1}$ Dipartimento di Matematica e Applicazioni, Università di Napoli "Federico II", 80126 Napoli, Italy \\ ${ }^{2}$ Dipartimento di Fisica, Università di Napoli "Federico II", 80126 Napoli, Italy \\ ${ }^{3}$ INFN, Sezione di Napoli, 80126 Napoli, Italy
}

\begin{abstract}
We show that under appropriate conditions the impact of a very short and intense laser pulse onto a plasma causes the expulsion of surface electrons with high energy in the direction opposite to the one of propagation of the pulse. This is due to the combined effects of the ponderomotive force and the huge longitudinal field arising from charge separation ("slingshot effect"). The effect should also be present with other states of matter, provided the pulse is sufficiently intense to locally cause complete ionization. An experimental test seems toi be feasible and, if confirmed, would provide a new extraction and acceleration mechanism for electrons, alternative to traditional radio-frequencybased or Laser-Wake-Field ones.
\end{abstract}

\section{INTRODUCTION}

Recently developed laser technologies have allowed the production of very intense and coherent electromagnetic (EM) waves concentrated in ultra-short pulses, whose interaction with matter is characterized by fast, huge and highly nonlinear effects. In particular, the very strong longitudinal ponderomotive forces caused by such laser pulses produces very large and very fast charge separations between ions and electrons and thereby very strong longitudinal electric fields (in matter other than plasma, this occurs after the huge kinetic energy transfered to the electrons has locally ionized matter into a plasma). In some conditions, a plasma wave with a phase velocity almost equal to the group velocity of the laser pulse appears behind the latter. This characterizes a mechanism known as Laser Wake Field (LWF) excitation [1-3], which can be used to accelerate beams of charged particles injected from outside (external injection [4]) or belonging to the plasma itself (self injection [5]).

In plasma-based acceleration schemes working with the external injection, lasers with power of up to hundreds of Terawatts are employed. Their typical intensity and pulse duration range from $10^{20}$ to $10^{22}$ Watt $/ \mathrm{cm}^{2}$ and from sub-pico to femto-seconds, respectively. In these conditions, the ponderomotive effect leads to extreme charge separation corresponding to the maximum electric field $E_{\max }$ that can be achieved in a plasma of a given unperturbed density. This is reached when the electron density perturbation becomes of the order of the equilibrium density $n_{0}$ and $E_{\max }[\mathrm{V} / \mathrm{cm}] \approx \sqrt{n_{0}\left[\mathrm{~cm}^{-3}\right]}$. Then, for $n_{0} \sim 10^{18} \mathrm{~cm}^{-3}, E_{\max } \sim 1 \mathrm{GV} / \mathrm{cm}$. The ultra-strong acceleration gradients (compared to the maximum fields that are produced in the conventional accelerating machines based on radio-frequency cavities, which are of the order of tens $\mathrm{MV} / \mathrm{m}$ ) allow to reduce the length of the acceleration chamber of several orders of magnitude. Then, externally injected electrons could reach an energy of the order of a $1 \mathrm{GeV}$ in a few centimeters. However, the acceleration of an externally injected beam is effective only if the initial velocity of the latter is close to the phase velocity of the plasma wave (hence to the light speed $c$ ), so that the dragging action of the wake lasts sufficiently long. Therefore, acceleration machines based on external injection can be used only after some pre-acceleration stage.

With a very intense laser pulse, the plasma electrons reach very high energies in very short times. In the self injection scheme [5] the accelerated electrons are those which are naturally (albeit violently) ejected from the plasma itself. Actually, in the works of experimental feasibility, this second acceleration process, if compared to the external injection scheme, was not very efficient and reliable in terms of intensity, energy spread and collimation. Therefore, it appeared to be more suitable as an electron beam source and pre-acceleration device to be used before a subsequent acceleration stage, whether conventional or plasma-based. However, recent investigations [6, 7] have shown that acceleration schemes that are based on a violent laser-plasma interaction should work in a regime for which the laser pulse is strong enough to blow out electrons and leave a wake of immobile positively charged ions (bubble regime or blowout regime). Such a regime has been confirmed by several valuable particle-in-cell simulations. A bubble can both trap and accelerate the background electrons and it seems to be necessary to produce a very collimated, quasimonoenergetic, self-injected electron beam. Bubbles are typically encircled by a return current that is created by relativistic electrons. However, a reliable quantitative description is still missing. Remarkably, a very recent experiment with self-injection has produced quasimonoenergetic electrons with energy well beyond $1 \mathrm{GeV}$. By applying new Petawatt laser technology, a group of researchers of Texas University at Austin [8] was able to produce electron bunches with a spectrum prominently peaked at $2 \mathrm{GeV}$ with only a few percent energy spread and unprecedented sub-milliradian divergence. This circumstance seems to characterize the signature of the bubble formation that is followed immediately by a strong localized injection.

In this paper, we show that a different acceleration 
mechanism of plasma electrons may occur under suitable conditions for the pulse length, duration and shape. In the self injection scheme the plasma electrons are dragged in and ejected forward, whilst in this new slingshot mechanism surface electrons (i.e. plasma electrons in a thin layer just beyond the surface of the plasma) are expected to be expelled with high energy backwards. This means that, shortly after the impact of a suitable ultra-short and ultra-intense laser pulse in the form of a pancake normally onto a plasma, such electrons are expelled along the direction that is opposite to the one of the pulse propagation. The mechanism is very simple: the surface electrons first are given sufficient electric potential energy by displacement (through the ponderomotive force produced by the pulse) with respect to the ions, then are pulled back by the electric force exerted by the latter and the other electrons, and leave the plasma; provided the spot size is sufficiently small their energy will be enough to escape to infinity. The stages are schematically depicted in fig. 1. Below we show that the conditions for this to happen are that the pulse is sufficiently short, the radius $R$ of the pancake is sufficiently small for the EM field inside the pulse to be sufficiently intense, but also relatively large to avoid trapping of the electrons. As this is based only on the interaction of the pulse with the first layer of plasma, a reliable, rather explicit magnetohydrodynamic description seems to be possible at least for a low density plasma. This is given here, referring the reader to 9 for the proof of some essential mathematical results (a summary can be found in [10]). The results are used to suggest possible experiments at the Italian INFN facility FLAME (Frascati Laser for Acceleration and Multidisciplinary Experiments) [1], and preliminary estimates for possible experiments at more powerful facilities like the Extreme Light Infrastructure (ELI) [12. We expect that for higher density plasmas and suitably higher intensities of the EM field the slingshot effect still occurs and leads to the expulsion of higher energy electrons; a quantitative estimate of the latter is not possible by computations within the low density approximation considered in [9], and will be considered elsewhere.

It should be pointed out that the interaction of very intense laser radiation with the boundary of an overdense plasma has been studied in 13 15] using particle-in-cell simulations or (simplified) analytical models. It was found that the effect on the EM radiation (respectively transmission and reflection, absorption, conversion from incident femtosecond to reflected attosecond pulses) may be accompanied [13, 14] by (temporary or final) emission of nanobunches of electrons backwards; this is the result of a multi-cycle in-out acceleration process (described stochastically, when the hydrodynamic description fails) of the boundary electrons by a quasistationary EM wave. On the contrary, the acceleration process presented here is induced by a short pulse onto an underdense plasma and produces a single bunch of electrons after a unique forward acceleration followed by a unique backward one; moreover, our description is purely hydrodynamic.

\section{THE MODEL}

We describe the plasma as a fully relativistic collisionless fluid, with the "plasma + EM field" system fulfilling the Lorentz-Maxwell and the continuity equations. We assume that the plasma is initially neutral, unmagnetized and at rest with electron density equal to zero and to a constant $n_{0}$ respectively in the regions $z<0$ and $z \geq 0$. We consider a purely transverse EM pulse in the form of a pancake with cylindrical symmetry around the $z$ axis, propagating in the positive $\hat{z}$ direction and hitting the plasma surface at $t=0$. Our simplified model of the impact leading to the slingshot effect consists of the following elements:

1. During the whole process we schematize the pancake pulse (see fig. 1-1) as a free plane pulse multiplied by the characteristic function of the cylinder $C_{R}$ of equation $\rho^{2} \equiv x^{2}+y^{2} \leq R^{2}$ (with some finite radius $R$ )

$\boldsymbol{E}^{\perp}(t, \boldsymbol{x})=\boldsymbol{\epsilon}^{\perp}(c t-z) \theta(R-\rho), \quad \boldsymbol{B}^{\perp}(t, \boldsymbol{x})=\hat{\boldsymbol{z}} \wedge \boldsymbol{\epsilon}^{\perp}(c t-z) \theta(R-\rho)$

$(\boldsymbol{E}, \boldsymbol{B}, c, \theta$ respectively stand for the electric and magnetic field, the velocity of light, the Heaviside step function, and we use CGS units throughout the paper); the 'pump' function $\boldsymbol{\epsilon}^{\perp}(\xi)$ vanishes outside some finite interval $0<\xi<l$. The pulse reaches the plasma surface $z=0$ at $t=0$. We justify (1) at the end of section IV.

2. We neglect the motion of ions (this is justified at the end of section III].

3. We first study (section II.1) the associated plane problem $[R=\infty$ in (1)] in a parameter range allowing simple approximations of the forward and backward motions of the surface electrons.

4. We determine (section II.2 a sufficiently large $R$ such that the approximation 3. is satisfactory for the surface electrons within some inner cylinder $C_{r}$ of equation $\rho^{2} \leq r^{2}$, with $r \geq R / 2$. We also estimate the corresponding deceleration of the electrons after expulsion and thus lower bounds for their number and final energy.

Items $1 \div 4$ and the other used approximations (see section IV make the effects of the model easily computable. Their justification consists in showing that their effect is either small or "conservative", i.e. that it leads to underestimate the size of the slingshot effect. This is fine for the purpose of the present work, which is to give a safe theoretical basis to the prediction of this new effect, rather than to optimize it. Most proofs and detailed calculations are concentrated in the appendix.

Now some remarks on notation. We denote as $\boldsymbol{x}_{e}(t, \boldsymbol{X})$ the position at time $t$ of the electrons' fluid element initially located at $\boldsymbol{X} \equiv(X, Y, Z)$, and for each fixed $t$ as $\boldsymbol{X}_{e}(t, \boldsymbol{x})$ the inverse. We denote as $n_{e}, \boldsymbol{v}_{e}$ the electrons' Eulerian density and velocity, and shall often use the dimensionless quantities $\boldsymbol{\beta}_{e} \equiv \boldsymbol{v}_{e} / c, \boldsymbol{u}_{e} \equiv \boldsymbol{p}_{e} / m c=$ $\boldsymbol{\beta}_{e} / \sqrt{1-\boldsymbol{\beta}_{e}^{2}}, \gamma_{e} \equiv 1 / \sqrt{1-\boldsymbol{\beta}_{e}^{2}}=\sqrt{1+\boldsymbol{u}_{e}^{2}}$. The Lagrangian counterparts depend on $t, \boldsymbol{X}$, rather than on $t, \boldsymbol{x}$, and are denoted by a tilde, e.g. $\widetilde{\boldsymbol{p}^{\perp}}(t, \boldsymbol{X})=\boldsymbol{p}^{\perp}\left[t, \boldsymbol{x}_{e}(t, \boldsymbol{X})\right]$. 


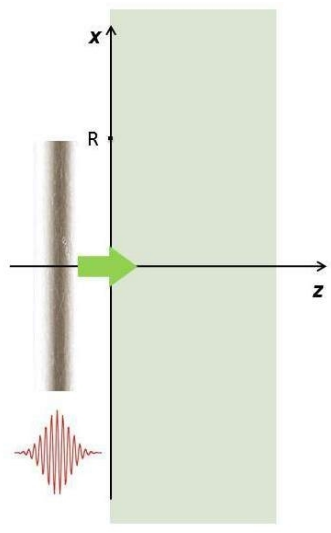

1. Free transverse EM wave + plasma at rest (plane idealization)

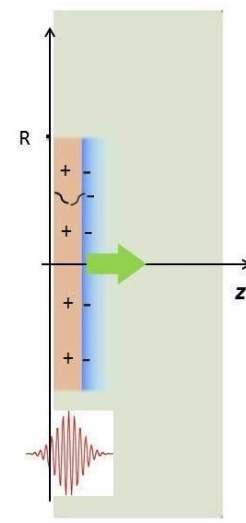

2. Boost by the ponderomotive force exerted by the EM wave (plane wave idealization)

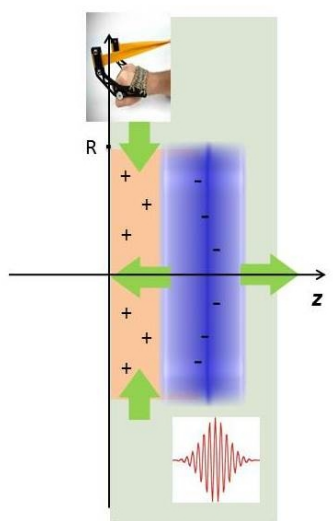

3. The electric force due to the separation of charges boosts the electrons backwards: like a SLINGSHOT (plane wave idealization)

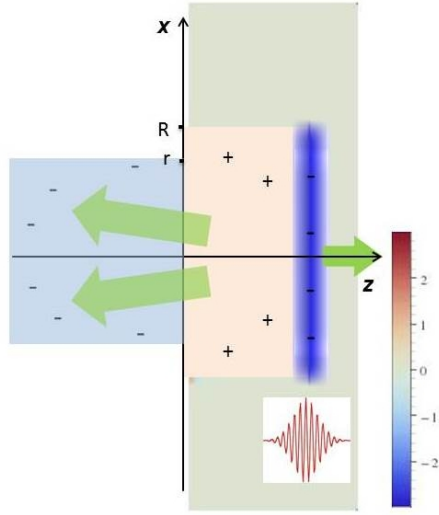

4. Since $R<\infty$, the Coulomb attraction by ions $\rightarrow 0$ as $z_{e} \rightarrow-\infty$, and allows $z_{e} \rightarrow-\infty$

FIG. 1. Schematic stages of the slingshot effect

\section{II.1. Plane wave idealization}

For $R=\infty$ we choose the gauge so that the transverse (with respect to $\hat{\boldsymbol{z}}$ ) vector potential is the physical observable $\boldsymbol{A}^{\perp}(t, z)=-\int_{-\infty}^{t} d t^{\prime} c \boldsymbol{E}^{\perp}\left(t^{\prime}, z\right)$; then $\boldsymbol{B}=\boldsymbol{B}^{\perp}=\hat{\boldsymbol{z}} \wedge \partial_{z} \boldsymbol{A}^{\perp}$, $c \boldsymbol{E}^{\perp}=-\partial_{t} \boldsymbol{A}^{\perp}$. By (1) it is $\boldsymbol{A}^{\perp}(t, z)=\boldsymbol{\alpha}^{\perp}(c t-z)$, where $\boldsymbol{\alpha}^{\perp}(\xi) \equiv-\int_{-\infty}^{\xi} d \xi^{\prime} \boldsymbol{\epsilon}^{\perp}\left(\xi^{\prime}\right)$, implying $\boldsymbol{\alpha}^{\perp}(\xi)=0$ for $\xi \leq 0$.

As known, the transverse component of the equation of motion $d \boldsymbol{p}_{e} / d t=-e\left(\boldsymbol{E}+\frac{\boldsymbol{v}}{c} \wedge \boldsymbol{B}\right)$ of the electrons implies $\boldsymbol{p}_{e}^{\perp}-\frac{e}{c} \boldsymbol{A}^{\perp}=$ const on the trajectory of each electron; this is zero at $t=0$, hence $\boldsymbol{p}_{e}^{\perp}=m c \boldsymbol{u}_{e}^{\perp}=\frac{e}{c} \boldsymbol{A}^{\perp}=\frac{e}{c} \boldsymbol{\alpha}^{\perp}$. The longitudinal component involves the electric force $F_{e}^{z} \equiv-e E^{z}$ and the magnetic one

$$
F_{m}^{z} \equiv-\frac{e}{c}\left(\boldsymbol{v}_{e} \wedge \boldsymbol{B}\right)^{z}=\frac{-e^{2}}{2 m c^{3} \gamma_{e}} \partial_{z} \boldsymbol{A}^{\perp 2}=\frac{e^{2}}{2 m c^{4} \gamma_{e}} \partial_{t} \boldsymbol{\alpha}^{\perp 2}(c t-z) .
$$

Regarding ions as immobile, the Maxwell equations imply

$$
E^{z}(t, z)=4 \pi e n_{0}\left\{z \theta(z)-Z_{e}(t, z) \theta\left[Z_{e}(t, z)\right]\right\} .
$$

(see e.g. eq. (46) in [9]). The electric force $\widetilde{F_{e}^{z}}(t, Z)=$ $-e \widetilde{E^{z}}(t, Z)=-e E^{z}\left[t, z_{e}(t, Z)\right]$ acting at time $t$ on the electrons initially located at $\boldsymbol{X}$ (with $Z \geq 0$ ) is therefore

$$
\widetilde{F_{e}^{z}}(t, Z)=\left\{\begin{array}{cc}
-4 \pi n_{0} e^{2} \Delta z_{e} \text { (elastic force) } & \text { if } z_{e}>0 \\
4 \pi n_{0} e^{2} Z \text { (constant force) } & \text { if } z_{e} \leq 0
\end{array}\right.
$$

where $\Delta z_{e} \equiv z_{e}(t, Z)-Z$ is the electron displacement with respect to its initial equilibrium position $Z$. $\widetilde{F_{e}^{z}}$ is thus the force of a harmonic oscillator (with equilibrium at $z_{e}=Z$ ) in the bulk and a nonnegative constant outside.

Assume that $\boldsymbol{\epsilon}^{\perp}=\epsilon_{s} \boldsymbol{\epsilon}_{o}^{\perp}$, where $\epsilon_{s}(\xi) \geq 0$ slowly varies inside the interval $0<\xi<l$ and is zero outside, while $\boldsymbol{\epsilon}_{o}^{\perp}(\xi)$ sinusoidally oscillates around zero with a period $\lambda \ll l$. If the pump $\boldsymbol{\epsilon}^{\perp}$ is very large, by continuity we expect the EM field to remain close to the compact-support travelling-wave (1) also for small $t>0$. In Ref. [9] it is shown that this is indeed the case in the space-time region $0 \leq c t-z \leq \xi_{0}, 0 \leq c t+z \ll \frac{2 \pi}{K \lambda}$, where $\xi_{0}$ is the first maximum point of $\epsilon_{s}(\xi)$, and $K \equiv \pi e^{2} n_{0} / m c^{2}$.

The first effect of the impact of the pulse is to boost all the electrons reached not only in the $x, y$ directions through the electric force $-e \boldsymbol{E}^{\perp}=-e \boldsymbol{\epsilon}^{\perp}$, but also in the $z$ direction through $F_{m}^{z}$. Since $\boldsymbol{p}_{e}^{\perp}=\frac{e}{c} \boldsymbol{\alpha}^{\perp}$ oscillates about zero for $0 \leq \xi \leq l$, and $\boldsymbol{\alpha}^{\perp}(\xi)=$ const $\simeq 0$ for $\xi \geq l$, then the transverse motion approximately averages to zero, and $\boldsymbol{p}_{e}^{\perp} \simeq 0$ after the pulse. Since $\boldsymbol{\alpha}^{\perp 2}$ is zero at the front of the pulse and positive inside, its time derivative is positive at the front; hence the initial $z$-boost is necessarily positive. Because of this boost, the first layer of ions remains unshielded while electrons accumulate just beyond the surface $S_{0}(t)$ of discontinuity of $n_{e}$ delimiting the $Z=0$ surface electrons [the resulting charge distribution in the approximation A9 to be considered here is depicted in the first four upper pictures of fig. 8. This charge separation generates the slingshot, i.e. the longitudinal electric force $\widetilde{F_{e}^{z}}$ pushing the boosted electrons backwards. If the time scale of significant variations of $\epsilon_{s}$ (and the duration $l / c$ of the pulse, a fortiori) is much larger than the characteristic period $\tau \gtrsim T_{H}^{n r}$ of oscillation of the electrons $\left(T_{H}^{n r} \equiv \sqrt{\pi m / n_{0} e^{2}}=2 \pi / \omega_{p}\right.$ is the period of the corresponding nonrelativistic harmonic oscillator) [16], then the electrons do many oscillations during the pulse, the power $P \equiv \widetilde{F_{m}^{z}} \widetilde{v^{z}}$ transfered by the magnetic force to each electron oscillates about zero, and its time integral $\mathcal{E}_{p}$ (the transfered energy, or slingshot loading) is approximately zero. This was the situation normally encountered in laboratories until a few years ago, because of the inability to generate sufficiently low densities and short pulses. If $l / c<T_{H}^{n r} / 4$, then $\widetilde{v^{z}}$ is positive during all the pulse while $\widetilde{F_{m}^{z}}$ oscillates about zero, and overall we obtain only a moderate slingshot loading $\mathcal{E}_{p}>0$ (if $l / c \ll T_{H}^{n r}$ a good approximation of the motion is the zerodensity solution recalled below). Therefore to increase $\mathcal{E}_{p}$ 
we study the range $l / c \sim T_{H}^{h r}$. Denoting as \langle\rangle the average over a period $\lambda$, we find $F_{p}^{z} \equiv\left\langle F_{m}^{z}\right\rangle \propto \partial_{t} \epsilon_{s}^{2}(c t-z)$, and the ponderomotive force $F_{p}^{z}$ is respectively positive, negative when $\epsilon_{s}^{2}$ is strictly increasing, decreasing - as known. If for simplicity $\epsilon_{s}^{2}(\xi)$ has a unique maximum point $\xi_{0}$, to maximize $\mathcal{E}_{p}$ we should make $\widetilde{v^{z}}$ switch from positive to negative only once during the pulse, exactly at the time $\bar{t}$ when the maximum reaches the electrons, so that $\langle P\rangle \simeq F_{p}^{z} \widetilde{v^{z}}$ keeps nonnegative during all the motion. This can be achieved by tuning $n_{0}$ and the pulse length in the range

$$
l \sim c T_{H}^{n r} / 2=\sqrt{\pi m c^{2} / 4 n_{0} e^{2}},
$$

as well as the shape of the pulse 17. After overcoming the surface electrons, the EM pulse will further propagate forward, slowly damped; it may also generate a wake with deeper electrons in the plasma. We shall ignore such phenomena and follow only the motion of the surface electrons, showing that a thin layer is finally expelled with high energy in the backward direction.

The above picture is confirmed by the first steps of an iterative resolution scheme of the plane problem proposed in 9. The initial step is the 'zero-density' solution, which we summarize in eq. A1 A3). According to it, the longitudinal motion of any electron is never backward. The $Z=0$ electrons are reached by the maximum of $\epsilon_{s}^{2}$ at time $t_{0}=\Xi_{e}\left(\xi_{0}\right) / c$, where $\Xi_{e}$ is defined in (A4). The error with respect to the real solution increases with $t$. In the second step (first correction to the 'zero-density' motion) these electrons invert their motion at a time $\bar{t}_{1}$ given in subsection A. Electrons initially located at small $Z>0$ will approximately move in the same way, after the space-time displacement $(Z, Z / c)$. For technical simplicity in this work we stick to parameters in a range [see conditions (A6) and the paragraph of eq. (A7)] such that the relative difference between the zero-density and the first corrected motion keeps much smaller than 1 for $t \leq t_{0}$ and rapidly grows for $t>t_{0}$, so that $\bar{t}_{1}-t_{0}$ is positive, but as small as possible. This ensures $[9$ that: i) the 'zero-density' motion A1 A2 is a good approximation of the real forward motion for all electrons initially located in a thin superficial layer, in particular it can be used to estimate their maximal displacement $\zeta$ :

$$
\zeta \simeq Y_{e}^{z}\left(\xi_{0}\right), \quad Y_{e}^{z}(\xi) \equiv \frac{e^{2}}{2 m^{2} c^{4}} \int_{0}^{\xi} d y \boldsymbol{\alpha}^{\perp 2}(y)
$$

ii) in this approximation the slingshot loading is efficient.

In the backward motion of the surface electrons for simplicity we underestimate as $\widetilde{F_{e}^{z}}(t, Z)$ the longitudinal force, neglecting the backward $\widetilde{F_{m}^{z}}$ due to the last part of the pulse and of the generated 'reflected' EM wave (see the final section). We denote the potential energy associated to the conservative force $F_{e}^{z}\left(z_{e}, Z\right) \equiv$ $4 \pi e^{2} n_{0}\left[Z-z_{e} \theta\left(z_{e}\right)\right]$ of $(3)$ as 18

$$
U\left(z_{e}, Z\right)=2 \pi n_{0} e^{2}\left[\theta\left(z_{e}\right) z_{e}^{2}-2 z_{e} Z+Z^{2}\right] .
$$

In fig. 2-left we plot $f \equiv F_{e}^{z} / 4 \pi e^{2} n_{0}$ and $u \equiv U / 2 \pi e^{2} n_{0}$ as functions of $z_{e}$ for a few values of $Z \geq 0$. Using energy conservation during the backward motion

$$
\begin{aligned}
H \equiv m c^{2} \gamma_{e}\left(z_{e}, Z\right)+U\left(z_{e}, Z\right) & =m c^{2} \sqrt{1+\boldsymbol{u}_{e i}^{\perp 2}}+2 \pi n_{0} e^{2} \zeta^{2} \\
& \geq m c^{2}+2 \pi n_{0} e^{2} \zeta^{2}
\end{aligned}
$$

one can compute $\gamma_{e} \equiv 1 / \sqrt{1-\boldsymbol{v}_{e}^{2} / c^{2}}$, and therefore also $\beta_{e}^{z}$, as functions of $z_{e}, Z$ (here $m c \boldsymbol{u}_{e i}^{\perp}$ is the electron transverse momentum when the displacement is maximal, $z_{e}-Z=\zeta$ ), and the longitudinal motion by quadrature (appendix A); the combined forward-backward motion is summarized in eq. (A9) and plot in fig. 7 for some fixed, relevant values of the parameters and a few different values of $Z$ (see below); as the corresponding map $Z \mapsto z$ is one-to-one for all $t$, different worldlines do not intersect. This shows that in this time lapse our treatment of the electron fluid as collisionless is consistent. The mechanical energy of the $Z=0$ electrons after expulsion $\left(z_{e}<0\right)$ is purely kinetic, because $U\left(z_{e}, 0\right)=0$. However, as $U(-\infty, Z)=\infty$ for $Z>0$, one concludes that in the $R=\infty$ idealization only the former electrons escape to $z_{e}=-\infty$ infinity; inner electrons invert their motion where $\gamma_{e}$ reaches its minimum $\simeq 1$ and then oscillate around $Z$. We now show that $R<\infty$ allows the escape of a thin layer of electrons.

\section{II.2. Inclusion of 3D-effects}

In the realistic case of a finite radius $R$ the ponderomotive force of the pulse will boost only the electrons approximately in the cylinder $C_{R}$ of the same radius. We require $R$ to be sufficiently large with respect to $\zeta$ in order that: i) the electrons in a cylinder $C_{r}$ of radius $r>0$ undergo approximately the same motion (A9) as with $R=\infty$; ii) there is no trapping, i.e. the way backwards out for the surface electrons within $C_{r}$ is not obstructed by the electrons initially located just outside the lateral surface $\partial C_{R}$ of $C_{R}$ (the latter electrons first are boosted outward, because on $\partial C_{R}$ so is directed the gradient of $\boldsymbol{E}^{\perp 2}$ of the pulse, then move towards the $z$-axis attracted by the ions). At the end of appendix B we show that both requirements are satisfied by the condition

$$
R \gtrsim \sigma \zeta
$$

with $\sigma$ a little larger than 1 (at least in the range of relevant conditions). We now show that: iii) due to the finite $R$, outside the bulk the attracting force by the ions decreases sufficiently fast with $\left|z_{e}\right|$ to allow a thin layer of the expelled electrons to escape to infinity.

As said $\widetilde{\boldsymbol{p}_{e}^{\perp}} \simeq 0$ after the pulse. We consider only the motion of the electrons moving along the $\vec{z}$-axis $(\rho=0)$ after the pulse; in other words we consider those electrons which experience the strongest restoring force after expulsion, by symmetry reasons. In fig. 3-up we depict the expected charge distribution of the electrons initially 

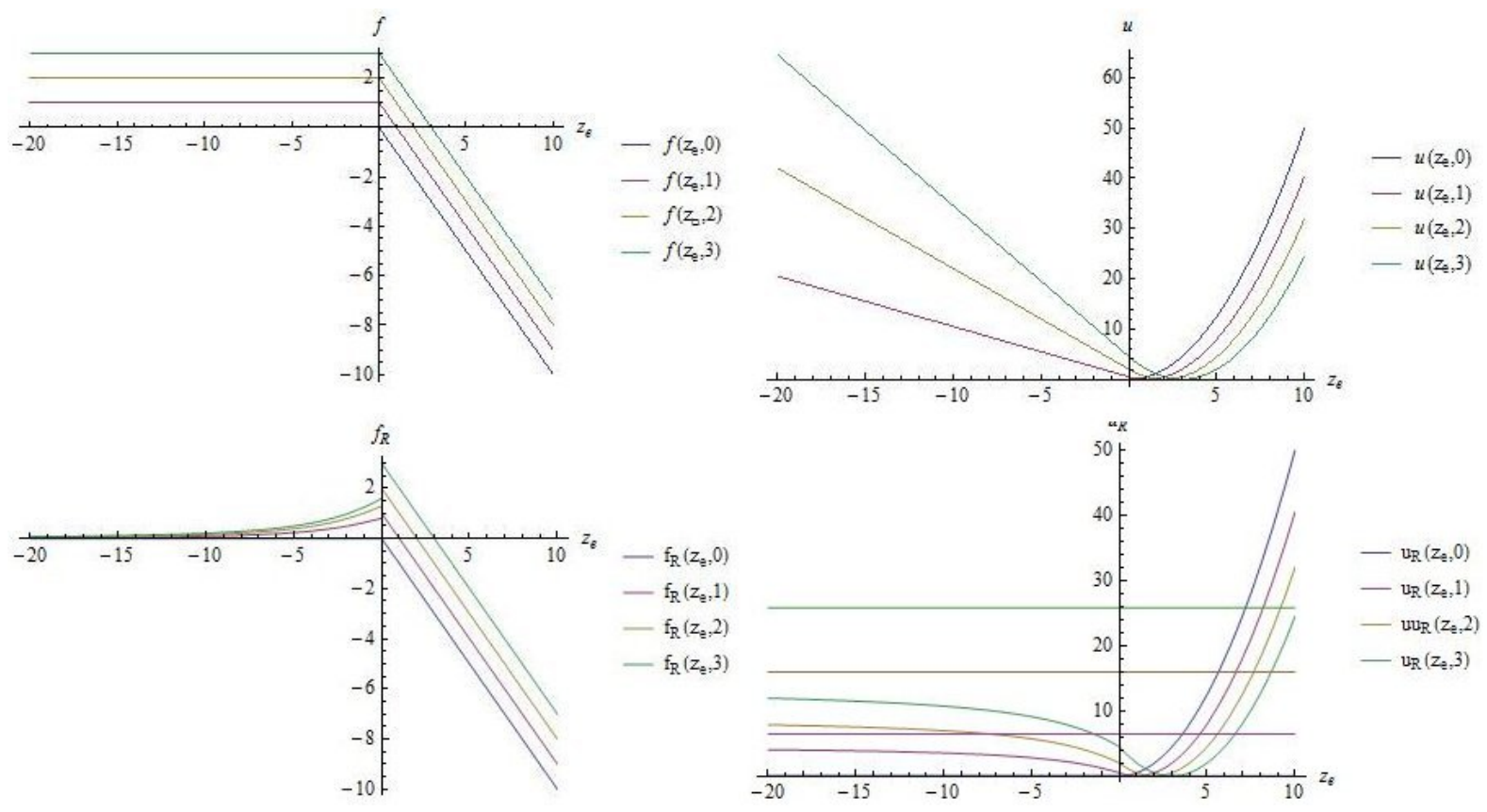

FIG. 2. The rescaled longitudinal electric force $f$ (left, up) and the associated rescaled potential energy $u$ (left, down) in the idealized plane wave case, the rescaled longitudinal electric force $f_{R}$ (right, up) and the associated rescaled potential energy $u_{R}$ (right, down) in the case of a pancake of radius $R=5$, plotted as functions of $z_{e}$ for $Z=0,1,2,3$; the horizontal lines in the right down graph are the left asymptotes of $u$ for $Z=0,1,2,3$.

located at $Z \gtrsim 0$ at a time $t$ shortly after their expulsion. The light blue area is occupied only by electrons. The left border, the dashed line and the solid line respectively represent the surfaces $S_{0}, S_{1}, S_{2}$ occupied by the electrons initially located at the points $\boldsymbol{X}^{\prime} \in C_{r}$ such that $Z^{\prime}=0, Z, 2 Z$. The orange area is positively charged due to an excess of ions. We can bound the real electric longitudinal force $\widetilde{F_{e}^{z r}}$ experienced by the electrons moving along the $\vec{z}$-axis as follows:

$$
0 \leq \widetilde{F_{e}^{z r}}(t, Z)=-e \widetilde{E_{-}^{z}}(t, Z)-e \widetilde{E_{+}^{z}}(t, Z) \leq F_{e R}^{z}\left[z_{e}(t, Z), Z\right]
$$

Here $\widetilde{E_{-}^{z}}(t, Z)$ stands for the part of the longitudinal electric field generated by the electrons between $S_{0}, S_{2}$; since those between $S_{0}, S_{1}$ have by construction the same charge as those between $S_{1}, S_{2}$, but are more dispersed, it will be $-e \widetilde{E_{-}^{z}}(t, Z) \leq 0$. The part $-e \widetilde{E_{+}^{z}}(t, Z)$ of the longitudinal electric force generated by the ions and the remaining electrons (at the right of $S_{2}$ ) will be smaller than the force $F_{e R}^{z}$ generated by the charge distribution depicted in fig. 3 down, where the remaining electrons are located farther from $\left(0,0, z_{e}\right)$ (not in their actual positions, but in their initial ones $\boldsymbol{X}^{\prime}$ ) and therefore generate a smaller repulsive force. Using cylindrical coordinates $\left(Z^{\prime}, \rho^{\prime}, \varphi^{\prime}\right)$ for $\boldsymbol{X}^{\prime}$ one easily finds for $z_{e} \leq 0$

$$
\begin{aligned}
& F_{e R}^{z}\left(z_{e}, Z\right) \equiv \int_{0}^{2 Z} d Z^{\prime} \int_{0}^{R} d \rho^{\prime} \frac{2 \pi n_{0} e^{2} \rho^{\prime}\left(Z^{\prime}-z_{e}\right)}{\left[\rho^{\prime 2}+\left(Z^{\prime}-z_{e}\right)^{2}\right]^{3 / 2}} \\
& =2 \pi n_{0} e^{2}\left[2 Z+\sqrt{z_{e}^{2}+R^{2}}-\sqrt{\left(2 Z-z_{e}\right)^{2}+R^{2}}\right] .
\end{aligned}
$$
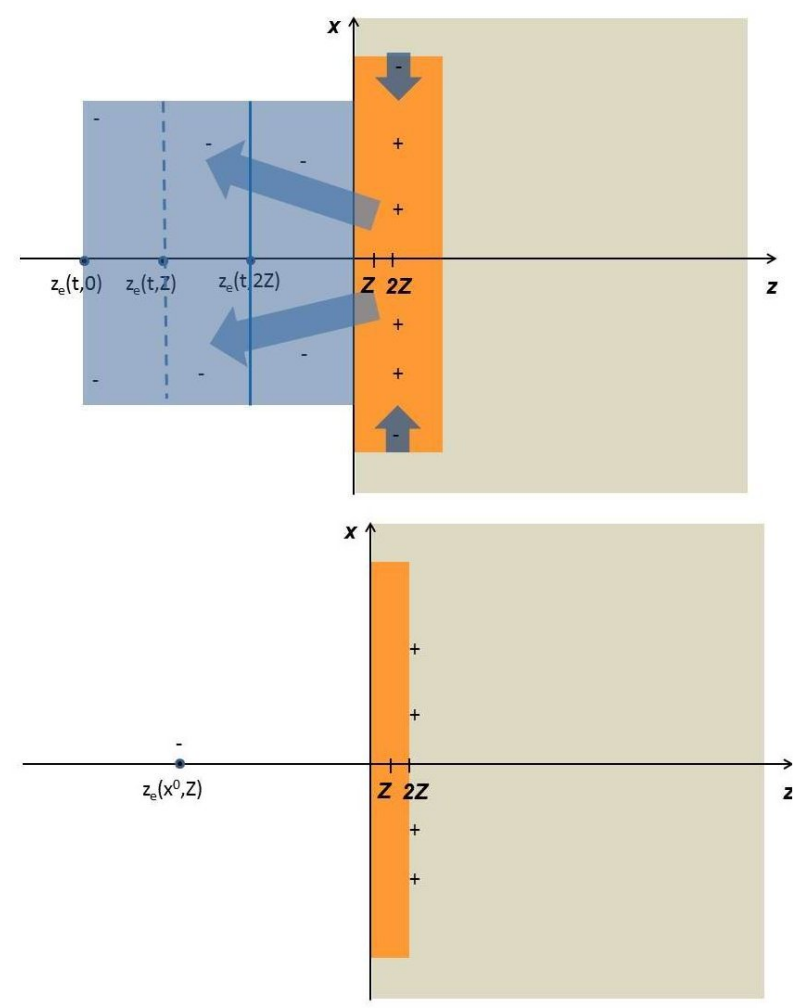

FIG. 3. Up: schematic picture of the expected charge distribution shortly after the expulsion (long arrows) of surface electrons; short arrows represent the inward motion of lateral electrons. Down: simplified charge distribution. 

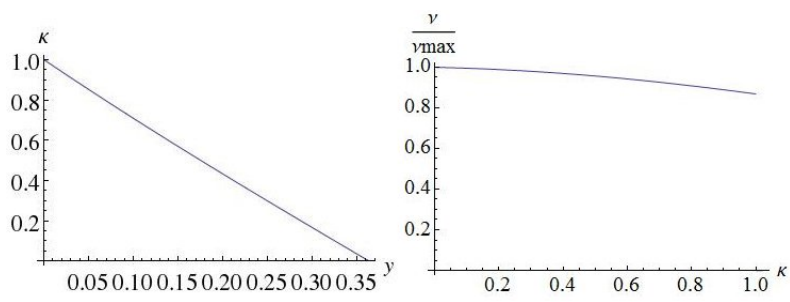

FIG. $4 . \quad \kappa(y)$ vs. $y \in\left[0, y_{M}\right] \quad\left(y_{M} \equiv Z_{M} / \zeta\right)$ (left), and the kinetic energy spectrum $\nu(\kappa)$ [in units of $\left.\nu_{\max }=\nu(\kappa=1)\right]$ vs. $\kappa \in[0,1]$ (right), for $R=1.5 \zeta$.

$F_{e R}^{z}$ is nonnegative and goes to zero as $z_{e} \rightarrow-\infty$, as it must be; it becomes a function of $t$ through $z_{e}(t, Z)$ only. The associated potential energy is 19

$$
\begin{aligned}
U_{R}\left(z_{e}, Z\right)= & \pi n_{0} e^{2}\left[\left(z_{e}-2 Z\right) \sqrt{\left(z_{e}-2 Z\right)^{2}+R^{2}}-4 Z z_{e}+\right. \\
& R^{2} \sinh ^{-1} \frac{z_{e}-2 Z}{R}-z_{e} \sqrt{z_{e}^{2}+R^{2}}-R^{2} \sinh ^{-1} \frac{z_{e}}{R} \\
& \left.+2 Z^{2}+2 Z \sqrt{4 Z^{2}+R^{2}}+R^{2} \sinh ^{-1} \frac{2 Z}{R}\right],
\end{aligned}
$$

a decreasing function of $z_{e}$ with finite left asymptotes for any $Z \geq 0$. In fig. 2-right the plots of $f_{R} \equiv F_{e R}^{z} / 4 \pi n_{0} e^{2}$, $u_{R} \equiv U_{R} / 2 \pi n_{0} e^{2}$ replace those of $f, u$ for $z_{e} \leq 0$. Denote as

$$
\begin{aligned}
& \gamma_{e}^{\infty}(Z)=1+\left[2 \pi n_{0} e^{2} \zeta^{2}-U_{R}(-\infty, Z)\right] / m c^{2} \\
& \quad=1+K\left[2 \zeta^{2}+2 Z^{2}-2 Z \sqrt{4 Z^{2}+R^{2}}-R^{2} \sinh ^{-1} \frac{2 Z}{R}\right]
\end{aligned}
$$

the $z_{e} \rightarrow-\infty$ limit of the relativistic factor $\gamma_{e}$ determined by replacing $U$ by $U_{R}$ with $\boldsymbol{u}_{e i}^{\perp 2}=0$ in (7), and as $Z_{M}$ the value of $Z$ for which $\gamma_{e}^{\infty}(Z)=1$. As said, by such a replacement we overestimate the restoring rightwards force experienced by the electrons for $z_{e}<0$. Consequently, the real relativistic factor of the electrons will be larger than the above $\gamma_{e}$, and the $M_{0} \equiv \pi r^{2} Z_{M} n_{0}$ electrons in $C_{r}$ with $0 \leq Z \leq Z_{M}$ will be only part of those escaping to infinity; a lower bound for their final relativistic factor is $\gamma_{e}^{\infty}(Z)$. We find also the bound $|Q| \geq e \pi n_{0} r^{2} Z_{M}$ for the total expelled electric charge $Q$. In fig. 4-left we plot the normalized kinetic energy $\kappa \equiv\left[\gamma_{e}^{\infty}-1\right] / 2 K \zeta^{2}$ as a function of $y \equiv Z / \zeta$ for $R=1.5 \zeta$ (by definition $0 \leq \kappa \leq 1$, $0 \leq y \leq y_{M}$, where $\left.y_{M} \equiv Z_{M} / \zeta\right)$. The plot for $R=1.25 \zeta$ does not differ significantly. The fraction of expelled electrons with initial position $Z^{\prime} \in[Z, Z+d Z]$ is no less than $\pi r^{2} n_{0} d Z=\pi r^{2} \zeta n_{0} d y$ and, using $\gamma_{e}^{\infty}$ instead of the real final relativistic factor, the fraction with kinetic energy in the interval $[\kappa, \kappa+d \kappa]$ is no less than $\nu(\kappa) d \kappa$, where

$$
\nu(\kappa)=-\frac{\pi r^{2} \zeta n_{0}}{\left.\kappa^{\prime}(y)\right|_{y=\hat{y}(\kappa)}}=\frac{\pi r^{2} \zeta n_{0}}{2\left[\sqrt{4 y^{2}+R^{2} / \zeta^{2}}-y\right]_{y=\hat{y}(\kappa)}}
$$

$[\hat{y}(\kappa)$ is the inverse of $\kappa(y)]$ represents the associated energy spectrum; this is plotted in fig. 4 right. By 10 the final relativistic factor of the expelled electrons is

$$
\gamma_{e M} \equiv \gamma_{e}^{\infty}(0) \simeq 1+2 K \zeta^{2} \simeq 1+2 K\left[Y_{e}^{z}\left(\xi_{0}\right)\right]^{2} ;
$$

the last $\simeq$ holds under condition $\mathrm{A} 6$.

We expect $\zeta, \gamma_{e_{M}}$ to grow with the intensity - although at a slower rate - even if A6 is not fulfilled.

\section{EXPERIMENTAL FEASIBILITY}

We now briefly discuss the experimental conditions for the slingshot effect, in particular for possible experiments at the FLAME facility in Frascati (but several other laboratories have comparable lasers) and at future ELI infrastructures. Laser pulses of wavelength $\lambda$, length $l \gg \lambda$, for simplicity symmetric around $\xi_{0}=l / 2$, concentrated onto an area $\pi R^{2}$, have an energy

$$
\mathcal{E}=\int d V \frac{\boldsymbol{E}^{\perp 2}+\boldsymbol{B}^{\perp 2}}{8 \pi} \simeq \frac{(\pi R)^{2}}{\lambda^{2}} \int_{0}^{l} d \xi \boldsymbol{A}^{\perp 2}=\frac{4\left(\pi m c^{2} R\right)^{2}}{(e \lambda)^{2}} Y_{e}^{z}\left(\xi_{0}\right) .
$$

(we have used the relation $\boldsymbol{E}^{\perp} \simeq \boldsymbol{A}^{\perp} 2 \pi / \lambda$, valid for a modulated approximately monochromatic plane wave). By (5) $\zeta \simeq \mathcal{E}(e \lambda)^{2} / 4\left(m \pi c^{2} R\right)^{2}$, and (8) takes the form

$$
R^{3} \gtrsim \frac{\sigma \mathcal{E}(e \lambda)^{2}}{4\left(\pi m c^{2}\right)^{2}}
$$

To maximize $\zeta$ and $\gamma_{e M}$ we choose $R=\sigma \zeta$; we obtain

$$
\sigma \zeta=R=\left[\frac{\sigma \mathcal{E}(e \lambda)^{2}}{4\left(\pi m c^{2}\right)^{2}}\right]^{\frac{1}{3}} .
$$

The laser at the FLAME facility can shoot linearly polarized pulses with $\lambda \simeq 8 \times 10^{-5} \mathrm{~cm}$, energy $\mathcal{E}=5 \times 10^{7} \mathrm{erg}$, and an approximately gaussian modulating amplitude with width at half height $l^{\prime} \simeq 7.5 \times 10^{-4} \mathrm{~cm}$ [1, 20]. In the appendix we show that it is sufficient to choose $\sigma \equiv R / \zeta=$ $1.25,1.5$ to obtain the expulsion of the electrons in the corresponding layer $0 \leq Z \leq Z_{M}$ within a cylinder $C_{r}$ of radius $r \geq R / 2,2 R / 3$ respectively. By (5), (14) $\sigma=1.25$ gives $\zeta \simeq Y_{e}^{z}\left(\xi_{0}\right) \simeq 1.2 \times 10^{-3} \mathrm{~cm}, \quad R \simeq 1.5 \times 10^{-3} \mathrm{~cm}$, whereas $\sigma=1.5$ gives $\zeta \simeq Y_{e}^{z}\left(\xi_{0}\right) \simeq 1.07 \times 10^{-3} \mathrm{~cm}$, $R \simeq 1.61 \times 10^{-3} \mathrm{~cm}$. A plasma with $n_{0} \geq 10^{17} \mathrm{~cm}^{-3}$ is obtained by ionization from an ultracold gas (typically, helium) jet in a vacuum chamber hit by such an energetic laser pulse as soon as the Keldysh parameters $\Gamma_{i} \equiv \sqrt{U_{i} / \mathrm{k}}=\sqrt{2 U_{i} / m c^{2} u_{e}^{\perp 2}}$ for both first and second ionization become smaller than 1 ; here $\mathrm{k}$ is kinetic energy, and the potentials $U_{i}$ for first and second ionization are about $24 \mathrm{eV}, 54 \mathrm{eV}$ respectively. The length of the $z$ interval where both $\Gamma_{i}<1$ plays the role of pulse length $l$. The ionization is practically complete and immediate if $R \lesssim 13 \times 10^{-3} \mathrm{~cm}$, because for such field intensities the Keldysh parameter for double ionization reaches values $\Gamma_{i}^{d}<1 / 100 \ll 1$ [20, 21] very fast. In [9] it has been shown that the model predicts no significant difference if the modulating amplitude $\epsilon_{s}$ is not chosen as a gaussian (blue curve in fig. 5) but rather inside the support $0 \leq \xi \leq l_{p}$ as the following fourth degree polynomial

$$
\epsilon_{s}(\xi)=b_{p}\left[\frac{1}{4}-\left(\frac{\xi}{l_{p}}-\frac{1}{2}\right)^{2}\right]^{2} \theta\left[\frac{1}{4}-\left(\frac{\xi}{l_{p}}-\frac{1}{2}\right)^{2}\right]
$$

(purple curve in fig. 5), provided $b_{p}, l_{p}$ are chosen so that the widths at half height of $\epsilon_{s}^{2}$ and the pulse energy are the same; this gives $l_{p} \simeq 18.75 \times 10^{-4} \mathrm{~cm}$, corresponding 


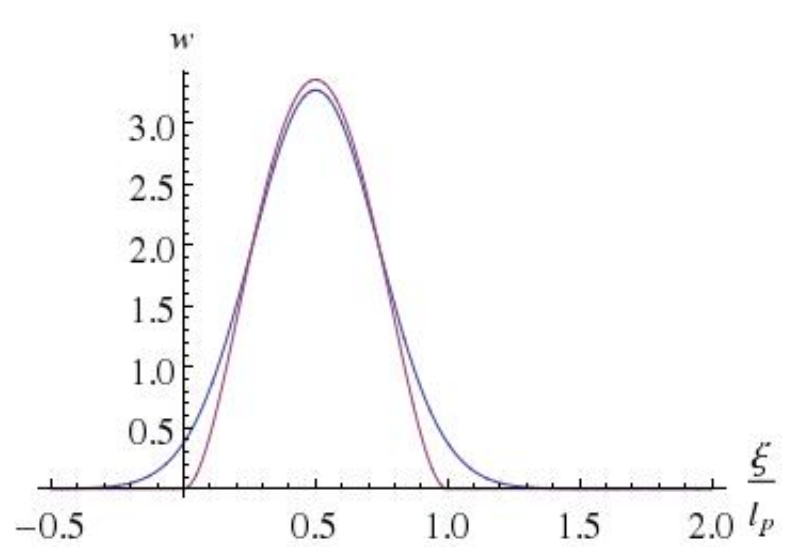

FIG. 5. Graphs of Gaussian (blue curve) and corresponding cut-off polynomial 15 (purple curve) normalized modulating amplitude $w(\xi) \equiv \frac{e \lambda}{2 \pi m c^{2}} \epsilon_{s}(\xi)$.

to a pulse duration of about $6.25 \times 10^{-14} \mathrm{~s}$. We use such polynomial modulating amplitude (15) rather than the Gaussian one to make numerical computations easier.

We adopt $K=2 l_{p}^{-2}$ as maximal $K$. This, replaced in the definition of $T(\xi)$, gives $T\left(\xi_{0}\right)=0.2$ and $T\left(\xi_{0}\right)=0.19$ if $\sigma \equiv R / \zeta=1.25, \sigma \equiv R / \zeta=1.5$ respectively; both fulfill A6 2 . So we adopt $K=2 l_{p}^{-2}$ as maximal $K$. Eq. 10 gives $Z_{M} \simeq 0.44 \zeta, Z_{M} \simeq 0.36 \zeta$; by (5), also condition $\mathrm{A} 6{ }_{1}$ is fulfilled in either case. The time $\bar{t}_{1}$ of inversion of the motion is very close to $t_{0}$ in either case.

In the central, right columns of table $\mathrm{I}$ we summarize these and other outputs respectively for $R / \zeta=1.25,1.5$.

As $n_{0} \ll n_{c}=\pi m c^{2} / e^{2} \lambda^{2} \simeq 1.7 \times 10^{21} \mathrm{~cm}^{-3}\left(n_{c}\right.$ is the critical density), we are indeed dealing with an underdense plasma. The pre-condition (4) for an efficient slingshot loading is fulfilled (the right-hand side $\pi / 2 \sqrt{K} \simeq$ $21 \times 10^{-4} \mathrm{~cm}$ is indeed of the order of the left-hand side). The maximal penetration $\zeta_{i}$ of the ions induced by the laser pulse is obtained from (5) replacing $m$ with their mass, hence $\zeta_{i} \ll \zeta$; similarly, the displacement of ions due to their mutual electric repulsion is much smaller than that of the lateral electrons (see appendix B). This justifies the description of ions as immobile.

\section{CONCLUSIONS AND FINAL REMARKS}

We have shown that the slingshot effect is possible if the laser pulse pancake is sufficiently short [eq. (4) and estimate after (A7)], its radius is not too small [eq. (8)], and the electromagnetic field inside is sufficiently intense for the expelled electrons to have a significant kinetic energy, i.e. a non-negligible $\gamma_{e_{M}}-1$. To make the latter condition quantitative and deduce conservative estimates of the final energy of the expelled electrons, as well as of the corresponding spectrum, we have used eq. (10, (12), (13), fig. 4, and assumed in addition the technical condition $\mathrm{A} 6 \mathrm{C}$.

\begin{tabular}{|l|c|c|}
\hline & $R / \zeta=1.25$ & $R / \zeta=1.5$ \\
\hline pulse energy $\mathcal{E}$ & $\simeq 5 \mathrm{~J}$ & $\simeq 5 \mathrm{~J}$ \\
wavelength $\lambda$ & $\simeq 8 \times 10^{-5} \mathrm{~cm}$ & $\simeq 8 \times 10^{-5} \mathrm{~cm}$ \\
pulse length $l_{p}$ & $\simeq 18.75 \times 10^{-4} \mathrm{~cm}$ & $\simeq 18.75 \times 10^{-4} \mathrm{~cm}$ \\
pulse spot radius $R$ & $=1.5 \times 10^{-3} \mathrm{~cm}$ & $=1.61 \times 10^{-3} \mathrm{~cm}$ \\
$K l_{p}^{2}$ & $=2$ & $=2$ \\
initial electron density $n_{0}$ & $=6.4 \times 10^{17} \mathrm{~cm}^{-3}$ & $=6.4 \times 10^{17} \mathrm{~cm}^{-3}$ \\
electrons' penetration $\zeta$ & $\simeq 1.2 \times 10^{-3} \mathrm{~cm}^{-3}$ & $\simeq 1.07 \times 10^{-3} \mathrm{~cm}^{-14}$ \\
time $\bar{t}$ of maximal penetration & $\simeq 7.15 \times 10^{-14} \mathrm{~s}$ & $\simeq 6.70 \times 10^{-14} \mathrm{~s}$ \\
time lapse of comeback $T_{H} / 4$ & $\simeq 5.247 \times 10^{-14} s$ & $\simeq 4.919 \times 10^{-14} \mathrm{~s}$ \\
time of expulsion $t_{e}$ & $\simeq 1.24 \times 10^{-13} \mathrm{~s}$ & $\simeq 1.162 \times 10^{-13} \mathrm{~s}$ \\
expelled layer thickness $Z_{M}$ & $\simeq 0.44 \zeta$ & $\simeq 0.36 \zeta$ \\
expelled electrons charge $|Q|$ & $\geq 1.41 \times 10^{-10} \mathrm{C}$ & $\geq 1.44 \times 10^{-10} \mathrm{C}$ \\
maximal relativistic factor $\gamma_{e M}$ & $\simeq 1.83$ & $\simeq 1.65$ \\
maximal expulsion energy $H$ & $\simeq 0.94 \mathrm{MeV}$ & $\simeq 0.85 \mathrm{MeV}$ \\
maximal electric field $E_{M}^{z}$ & $\simeq 1.4 \mathrm{GV} / \mathrm{cm}$ & $\simeq 1.25 \mathrm{GV} / \mathrm{cm}$ \\
\hline
\end{tabular}

TABLE I. Main inputs and outputs for FLAME experiments

Eq. $\mathrm{A}_{1}{ }_{1}$ in particular fixes un upper bound for $K$, hence for $n_{0}$. If we keep $R, \zeta$ fixed and decrease $n_{0}$ then A6 1 still holds and by (12) $\gamma_{e M}-1$ decreases proportionally. This scaling and the backward direction of expulsion may experimentally discriminate the slingshot effect from LWF or other acceleration mechanisms [22].

On the other hand, we expect that we can increase $\gamma_{e M}$ by decreasing $R$ and increasing $n_{0}$ so that $\zeta$ also decreases and (8) remains fulfilled. As this would violate the technical 9] condition (A6), a quantitative estimate of the corresponding slingshot effect is not possible at the level of approximation described here. Preliminary numerical computations at the next level indicate that the final kinetic energy of expelled electrons can be thus increased by at least one order of magnitude. This will be elaborated elsewhere.

The use of more powerful and shorter laser pulses would further increase the final energy of expelled electrons, while allowing the use of light solids as targets instead of gas jets. This could be accomplished, for instance, by the laser at the ELI facility, which will be able to shoot linearly polarized pulses with $\lambda \simeq 8 \times 10^{-5} \mathrm{~cm}$, energy $\mathcal{E} \simeq 10^{9} \mathrm{erg}$ and an approximately gaussian modulating amplitude with width at half height $l^{\prime} \simeq 3 \times 10^{-4} \mathrm{~cm}$ [12. Preliminary estimates seem to show that use of such a laser would allow to further increase the final energy of expelled electrons by more than one order of magnitude.

In the present model we have done a number of approximations. In addition to the ones already mentioned, we have also neglected the negative ponderomotive force of the pulse after its maximum has overcome the electrons, as well as the negative ponderomotive force of the 'reflected EM wave' generated by the impact of the pulse on the plasma; both add to the electrostatic force to in- 
crease the energy of the electrons in the expulsion phase. We have also approximated the transition region from $n_{e}=0$ to $n_{e}=n_{0}$ as the surface $z=0$, rather than a thin layer; we expect that the latter would modify the shape of the spectrum in fig. 4, but not the main results.

In (1) we have schematized the $\rho$-dependence of the pulse by $\theta(R-\rho)$, rather than by a more realistic smoothly decaying factor, such as $e^{-\rho^{2} / r^{2}(t)}$ with $r(0) \sim R$. Identifying $r(t)$ with $R$ is justified during the impact because $\zeta, Z_{M} \ll l_{R}$ (the Rayleigh length of the focalized laser pulse at FLAME is $l_{R} \sim 2 \times 10^{-2} \mathrm{~cm}$ ). The rather coarse approximation of a gaussian by a step function is adopted to ease the computations of the finite $R$ corrections (appendix $B$ and is a further reason why our prediction of the expelled charge $Q$ is an underestimate of the real one.

We have adopted all the decribed conservative estimates to give a safe basis to the prediction of this new effect, leaving optimization of the process as a task for further theoretical and experimental works.

\section{ACKNOWLEDGMENTS}

We thank L. Gizzi for information on FLAME and valuable suggestions, S. De Nicola for discussions. This work was partially supported by UniNA and Compagnia San Paolo under the grant "STAR Program 2013".

\section{Appendix A: Plane plasma equations}

Some results of [9]. Let $\widetilde{n_{e 0}}(Z) \equiv n_{e}(0, Z)$ be the initial density. In the $\widetilde{n_{e 0}} \equiv 0$ limit the electron motion in terms of the pump $\boldsymbol{A}^{\perp}(x)=\boldsymbol{\alpha}^{\perp}(c t-z)$ is:

$$
\begin{aligned}
& \boldsymbol{u}_{e}^{\perp}(t, z)=\frac{e \boldsymbol{\alpha}^{\perp}(c t-z)}{m c^{2}}, \quad u_{e}^{z}=\frac{1}{2} \boldsymbol{u}_{e}^{\perp 2}, \quad \gamma_{e}=1+u_{e}^{z}, \\
& z_{e}(t, Z)=c t-\Xi_{e}^{-1}(c t-Z) \\
& Z_{e}(t, z)=c t-\Xi_{e}(c t-z)=z-Y_{e}^{z}(c t-z), \\
& \boldsymbol{x}_{e}^{\perp}(t, \boldsymbol{X})=\boldsymbol{X}^{\perp}+\boldsymbol{Y}_{e}^{\perp}\left[c t-z_{e}(t, Z)\right] \\
& \boldsymbol{X}_{e}^{\perp}(t, \boldsymbol{x})=\boldsymbol{x}^{\perp}-\boldsymbol{Y}_{e}^{\perp}(c t-z), \\
& \partial_{t} Z_{e}=-c u_{e}^{z}, \quad \partial_{z} Z_{e}=\gamma_{e}, \quad \partial_{Z} z_{e}=1 / \widetilde{\gamma}_{e} .
\end{aligned}
$$

Relations (A1) describe in the Eulerian picture forward travelling-waves moving with phase velocity equal to $c$. In A2 we have used the primitives of $\boldsymbol{u}_{e}, \gamma_{e}$

$$
\boldsymbol{Y}_{e}(\xi) \equiv \int_{0}^{\xi} d y \boldsymbol{u}_{e}(y), \quad \Xi_{e}(\xi) \equiv \int_{0}^{\xi} d y \gamma_{e}(y)=\xi+Y_{e}^{z}(\xi) .
$$

As $u_{e}^{z} \geq 0, \quad Y_{e}^{z}(\xi)$ is increasing, $\Xi_{e}(\xi)$ is strictly increasing (and invertible), and the longitudinal motion is purely forward. Replacing A3 in the relations

$$
\begin{aligned}
& n_{e}(t, z)=\widetilde{n_{e 0}}\left[Z_{e}(t, z)\right] \partial_{z} Z_{e}(t, z), \\
& \widetilde{n_{e 0}}\left[Z_{e}(t, z)\right] \partial_{t} Z_{e}(t, z)+\left[n_{e} v_{e}^{z}\right](t, z)=0 .
\end{aligned}
$$

one obtains the Eulerian electron density in the next approximation. As said, here we assume $\widetilde{n_{e 0}}(Z)=n_{0} \theta(Z)$.

In Ref. 9] it is shown that the EM field remains close to the pump in the space-time region $0 \leq c t-z \leq \xi_{0}$, $0 \leq c t+z \ll \frac{2 \pi}{K \lambda}$, and that the motion A1 A4 is a good approximation of the real forward motion of all electrons with initial positions such that $0 \leq Z \leq Z_{M}$, provided [9]

$$
\begin{array}{ll}
\xi_{0}+2 Y_{e}^{z}\left(\xi_{0}\right)+2 Z_{M} \ll \frac{2 \pi}{K \lambda}, & T\left(\xi_{0}\right) \ll 1, \\
\text { where } \quad V(\xi) \equiv \int_{0}^{\xi} d y Y_{e}^{z}(y), & \\
G(\xi) \equiv \int_{0}^{\xi} d y \frac{\left(1+\boldsymbol{u}_{e}^{2}\right)\left[e^{8 K V}-1\right]}{1+\boldsymbol{u}_{e}^{2}+e^{8 K V}}(y), & T \equiv G / Y_{e}^{z} .
\end{array}
$$

$T\left(\xi_{0}\right)$ gives the relative error between $Y_{e}^{z}\left(\xi_{0}\right)$ and $\zeta$ in the next approximation.

According to formulae (33) and (61) of [9], in the next approximation the time when the surface electron invert their motion is $\bar{t}_{1}=\Xi_{e}\left(\xi_{1}\right) / c$, where $\xi_{1}$ is determined by $\exp \left[8 K V\left(\xi_{1}\right)\right]=1+\eta\left[e \lambda \epsilon_{s}\left(\xi_{1}\right) / 2 \pi m c^{2}\right]^{2} \eta=1,1 / 2$ respectively for circular, linear polarization) as a function of the physically tunable parameter $K$ (or, equivalently, of $n_{0}$ ). This equation can be solved for $K$ :

$$
K\left(\xi_{1}\right)=\ln \left\{1+\eta\left[e \lambda \epsilon_{s}\left(\xi_{1}\right) / 2 \pi m c^{2}\right]^{2}\right\} / 8 V\left(\xi_{1}\right)
$$

$\left[K\left(\xi_{1}\right)\right.$ is a strictly decreasing function from $K(0)=\infty$ to $K(l)=0] ; \quad K\left[\Xi_{e}^{-1}\left(c \bar{t}_{1}\right)\right]$ will be the value of $K$ leading to the specified inversion time $t_{1} . T(\xi) \ll 1$ for small $\xi$ and rapidly grows around $\xi_{1}$. We choose $\xi_{1}$ so that $\xi_{1}-\xi_{0}$ is positive, as small as possible, but makes $K\left(\xi_{1}\right)$ still compatible with A6; this guarantees $\bar{t} \simeq t_{0} \simeq \bar{t}_{1}$ and an efficient slingshot loading.

About the relativistic harmonic oscillator. Assuming for simplicity $\boldsymbol{u}_{e i}^{\perp}=0$, from (7) we find that $\Delta z(t, Z) \equiv z_{e}(t, Z)-Z$ fulfills

$$
\begin{aligned}
& 1+2 K\left[\zeta^{2}-\Delta z^{2}\right]=\tilde{\gamma}_{e}=1 / \sqrt{1-\tilde{\beta}_{e}^{z 2}} \Rightarrow \\
& \frac{1}{c} \frac{d \Delta z}{d t}=\tilde{\beta}_{e}^{z}=-\frac{\sqrt{4 K\left[\zeta^{2}-\Delta z^{2}\right]+4 K^{2}\left[\zeta^{2}-\Delta z^{2}\right]^{2}}}{1+2 K\left[\zeta^{2}-\Delta z^{2}\right]} \Rightarrow \\
& \Delta t=\frac{1}{2 c \sqrt{K}} \int_{\Delta z}^{\zeta} \frac{d y\left[1+2 K\left(\zeta^{2}-y^{2}\right)\right]}{\sqrt{\zeta^{2}-y^{2}+K\left(\zeta^{2}-y^{2}\right)^{2}}}
\end{aligned}
$$

Therefore the time lapse $\Delta t$ for the electrons to go from $z_{e}=Z+\zeta$ with zero initial velocity to $z_{e}=Z+\Delta z$ is

$$
\Delta t=\frac{\zeta}{c} f\left(\frac{\Delta z}{\zeta} ; K \zeta^{2}\right), \quad f(D ; \chi) \equiv \int_{D}^{1} \frac{d v\left[1 / 2+\chi\left(1-v^{2}\right)\right]}{\sqrt{\chi} \sqrt{1-v^{2}+\chi\left(1-v^{2}\right)^{2}}},
$$

$[\Delta z(t, Z)$ is independent of $Z$, and so is $\Delta t]$. In particular the time needed to return from $z=Z+\zeta$ to their initial equilibrium position $z=Z$ is, as claimed,

$$
\frac{1}{4} T_{H}=\frac{1}{2 c \sqrt{K}} \int_{0}^{\zeta} \frac{d z\left[1+2 K\left(\zeta^{2}-z^{2}\right)\right]}{\sqrt{\zeta^{2}-z^{2}+K\left(\zeta^{2}-z^{2}\right)^{2}}} .
$$



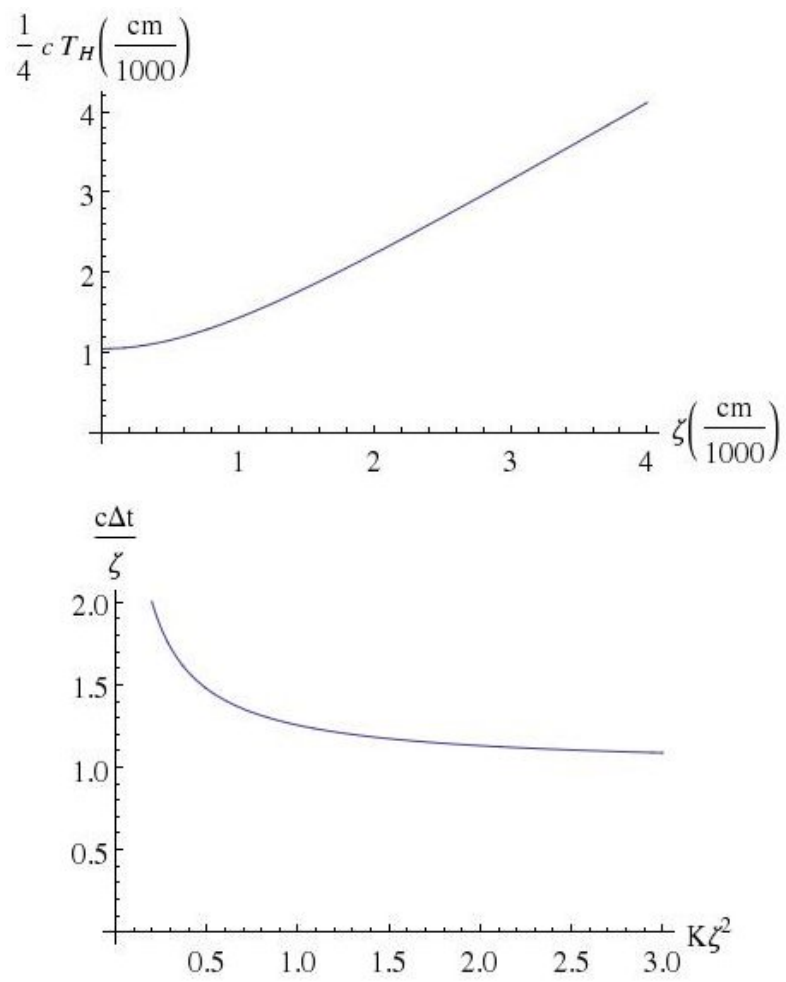

FIG. 6. The return time lapse $T_{H} / 4$ for $K \equiv \pi e^{2} n_{0} / m c^{2}=$ $5.7 \times 10^{-7} \mathrm{~cm}^{-2}$ as a function of the penetration $\zeta$ (up), the ratio $c T_{H} / 4 \zeta$ as a function of $K \zeta^{2}$ (down).

$T_{H}$ is the plasma oscillation period. In fig. 6 6 up we plot $c T_{H} / 4$ as a function of $\zeta$ for $K=5.7 \times 10^{-7} \mathrm{~cm}^{-2}$ (a value relevant for a possible experiment at FLAME, see below) 23]. The ratio $c T_{H} / 4 \zeta$ is a function of $K \zeta^{2}$ only, going to 1 as $K \zeta^{2} \rightarrow \infty$, see fig. 66-down, i.e. the average electron velocity goes to $c$ in this limit. The function $f$ defined in $\mathrm{A} 8 \mathrm{i}$ is a strictly decreasing, and therefore invertible, function of $D \in[-1,1]$, and so is

$$
m(D ; \chi) \equiv f(D ; \chi)-D .
$$

Inverting A8 at fixed $K \zeta^{2}$ we obtain $\Delta z=$ $f^{-1}\left(c \Delta t / \zeta, \overline{K \zeta^{2}}\right)$. Setting $\Delta t \equiv(t-\bar{t})-Z / c$ we obtain $z_{e}(t, Z)$ in the backward motion of the electrons. (A8) is also equivalent to $m\left(\Delta z / \zeta ; K \zeta^{2}\right)=c[(t-\bar{t})-z] / \zeta$; inverting the latter we find $Z_{e}(t, z)$ in the backward motion.

The whole electron motion until expulsion. Combining the last results with $\mathrm{A} 2$ we obtain our basic approximation for the whole motion of the electrons until their expulsion in the plane wave idealization:

$$
\begin{gathered}
z_{e}(t, Z)= \begin{cases}c t-\Xi_{e}^{-1}(c t-Z) & t \leq \bar{t}+Z / c \\
Z+\zeta f^{-1}\left\{\frac{c(t-\bar{t})-Z}{\zeta} ; K \zeta^{2}\right\} & t_{e} \geq t>\bar{t}+Z / c .\end{cases} \\
Z_{e}(t, z)= \begin{cases}z-Y_{e}^{z}(c t-z) & c t-z \leq \xi_{0} \\
z-\zeta m^{-1}\left\{\frac{c(t-\bar{t})-z}{\zeta} ; K \zeta^{2}\right\} & c t-z>\xi_{0} .\end{cases}
\end{gathered}
$$

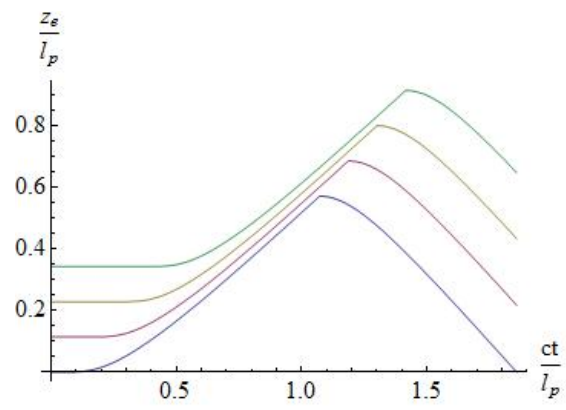

FIG. 7. The graphs of the function A9 of $t$ for the initial values $Z=0,0.2 \zeta, 0.4 \zeta, 0.6 \zeta$ and other parameters (pulse length $l_{p}$, etc.) given in the central column of Table I

In fig. (7) we plot the graphs of $z_{e}(\cdot, Z)$ (worldlines) normalized to $l_{p}$ (the pulse length available at FLAME) for a few fixed values of $Z$; these worldlines do not intersect, or equivalently the map $z \mapsto Z$ is one-to-one for each fixed $t$. Replacing $Z_{e}(t, z)$ in A5 we find $n_{e}(t, z)$.

\section{Appendix B: $R<\infty$ corrections to electron motions}

Here we study where A9 is a good approximation of the real electron motion induced by a pulse of finite $R$.

The general solution (retarded electromagnetic potential) of the Maxwell equation $\square A^{\mu}=4 \pi j^{\mu}$ in the Lorentz gauge $\left(\partial_{\mu} A^{\mu}=0\right)$ with zero asymptotic conditions reads

$$
A^{\mu}(t, \boldsymbol{x})=\int d^{3} x^{\prime} \frac{j^{\mu}\left[t_{r}\left(x, \boldsymbol{x}^{\prime}\right), \boldsymbol{x}^{\prime}\right]}{\left|\boldsymbol{x}-\boldsymbol{x}^{\prime}\right|}, \quad t_{r}\left(x, \boldsymbol{x}^{\prime}\right) \equiv t-\frac{\left|\boldsymbol{x}-\boldsymbol{x}^{\prime}\right|}{c},
$$

and $\boldsymbol{E}(t, \boldsymbol{x})=\frac{-1}{c} \partial_{t} \boldsymbol{A}-\nabla A^{0}$. As a first rough approximation of the real current induced by the pump (1) we adopt

$$
\left(j^{\mu}(t, \boldsymbol{x})\right) \equiv e\left(\widetilde{n_{e 0}}(z)-n_{e}(t, z),-\left(n_{e} \boldsymbol{\beta}_{e}\right)(t, z)\right) \theta(R-\rho),
$$

where $\widetilde{n_{e 0}}(Z)=n_{0} \theta(Z)$ and $n_{e}, \boldsymbol{\beta}_{e}$ are determined from A5, A9 A10; a plot of $j^{0}$ is in fig. 8. As velocities are relativistic, (B1) differs significantly from the instantaneous counterpart where $t_{r}$ is replaced by $t$. It gives the EM potential (and field, after derivation) generated by the electric current $(\bar{B} 2)$, which adds to the pump (1). We now estimate its influence over the electron motion (B2) itself, and where this can be neglected.

i) Determination of $\widetilde{E^{z}}$ on the $z$-axis. Below we show that if $\widetilde{n_{e 0}}(Z)$ vanishes at $Z<0$ the condition

$$
0 \leq z \leq c t \leq \sqrt{R^{2}+z^{2}}
$$

implies

$$
E^{z}(t, z \hat{\boldsymbol{z}})=4 \pi e\left\{\widetilde{N_{e}}(z)-\widetilde{N}_{e}\left[Z_{e}(t, z)\right]\right\},
$$

with $\widetilde{N}_{e}(Z) \equiv \int_{0}^{Z} d y \widetilde{n_{e 0}}(y)$ a primitive of $\widetilde{n_{e 0}}$. If $\widetilde{n_{e 0}}(Z)=$ $n_{0} \theta(Z)$ then $\widetilde{N}_{e}(Z)=n_{0} Z \theta(Z)$, and (B4) becomes (2), 

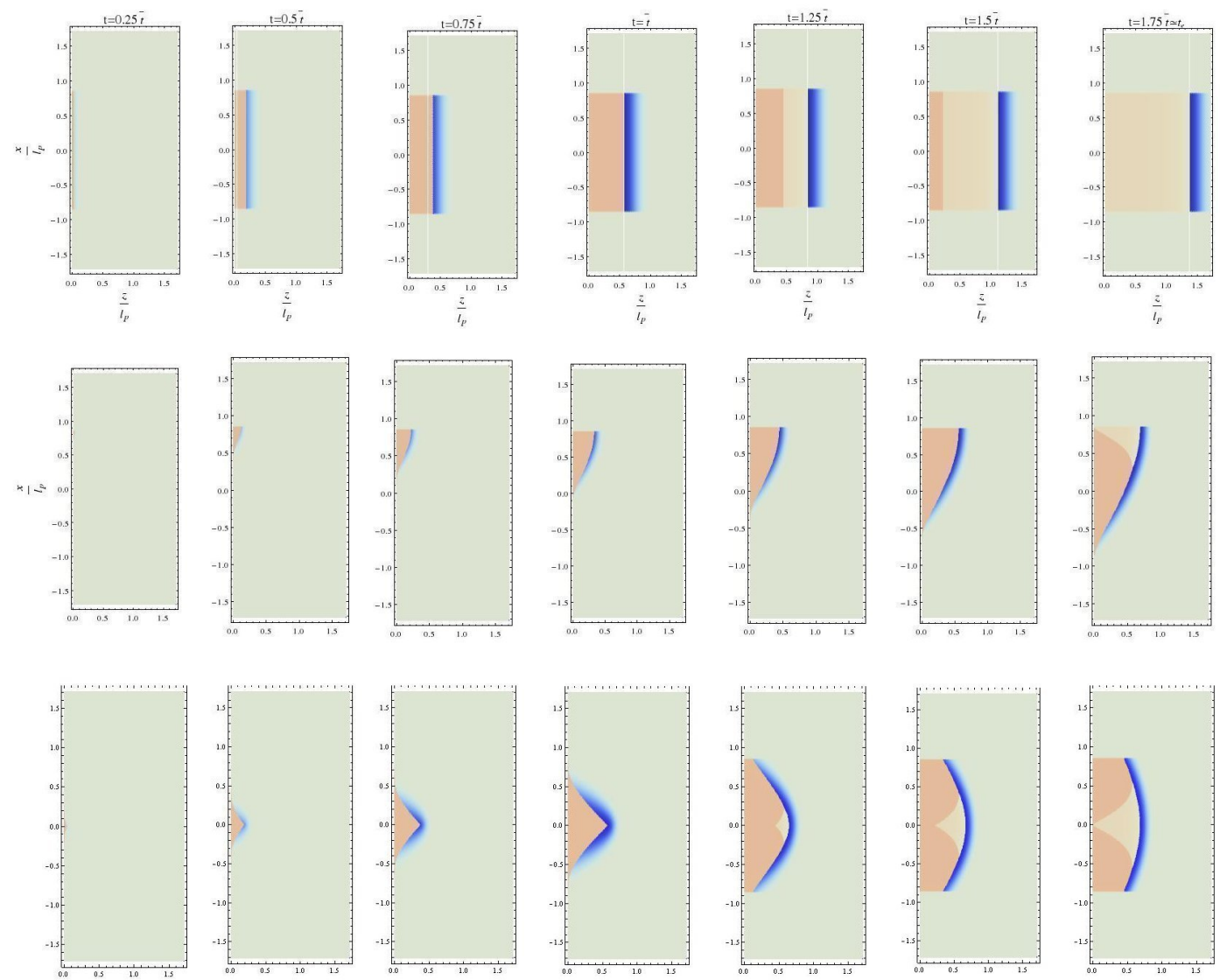

FIG. 8. Upper row: coloured plots in the $z, x$ plane of the normalized charge density $\frac{j^{0}(t, z, x)}{e n_{0}}$ multiplied by the characteristic function of the cylinder $C_{R}$, at $t=0.25 \bar{t}, 0.5 \bar{t}, 0.75 \bar{t}, 1.25 \bar{t}, 1.5 \bar{t}, 1.75 \bar{t}$ for the possible FLAME experiment parameters $R / \zeta=$ $1.5, K \zeta^{2}=0.654 ; j^{0}(t, z, x)$ is computed in the approximation A9. A10. $z, x$ are expressed in units of the length $l_{p}$ of the pulse. We name $S_{0}(t)$ the surface of discontinuity between the positively (brown) and negatively (blue) charged region.

Central row: corresponding coloured plots of the retarded normalized charge density $\frac{j^{0}\left(t_{r}, z\right)}{e n_{0}} \quad\left(t_{r}=t-\left|\boldsymbol{x}-\boldsymbol{x}^{\prime}\right| / c\right)$ at at the same times, as seen from the point $\boldsymbol{x}=R \hat{\boldsymbol{x}}$ of $\partial C_{R}$.

Lower row: corresponding coloured plots of the retarded normalized charge density $\frac{j^{0}\left(t_{r}, z x\right)}{e n_{0}} \quad\left(t_{r}=t-\left|\boldsymbol{x}_{e}(t, \mathbf{0})-\boldsymbol{x}^{\prime}\right| / c\right)$ at the same times, as seen from the point $\boldsymbol{x}_{e}(t, \mathbf{0})=z_{e}(t, 0) \hat{\boldsymbol{z}}$ on the $\vec{z}$-axis.

exactly as in the $R=\infty$ case. This should not come as a surprise: $(\mathrm{B} 3)$ ensures that the spacetime region where the $R<\infty$ and the $R=\infty 4$-current $j^{\mu}$ differ is not causally connected to the spacetime point $(c t, z \hat{\boldsymbol{z}})$ [24].

Moreover, we show that $\widetilde{E^{z}}(t, Z \hat{\boldsymbol{z}})$ remains very close to the Lagrangian counterpart of (2) for a time lapse growing with $R / \zeta$, even if the Lagrangian formulation of B3, i.e. $0 \leq z_{e}(t, Z) \leq c t \leq \sqrt{R^{2}+\left[z_{e}(t, Z)\right]^{2}}$, is not fulfilled. Tuning $R / \zeta$ we can thus neglect their difference until the expulsion time $t_{e}$. In particular we find that for the suggested experiments at FLAME $E^{z}(\bar{t}, \zeta \hat{\boldsymbol{z}}) / 4 \pi e n_{0}$ practically coincides with 1 with both choices $\sigma \equiv R / \zeta=$ $1.25,1.5$.

We determine $\widetilde{E^{z}}(t, Z \hat{\boldsymbol{z}})$ first for a generic $\widetilde{n_{e 0}}(Z)$ vanishing at $Z<0$. Denoting the cylindrical coordinates of $\boldsymbol{x}^{\prime}$ as $\left(z^{\prime}, \rho, \varphi\right)$, from $\mathrm{B} 1 \mathrm{~B} 2$ it follows

$$
\begin{aligned}
& \frac{1}{2 \pi e} E^{z}(t, z \hat{\boldsymbol{z}})=-\frac{1}{2 \pi e}\left[\frac{1}{c} \partial_{t} A^{z}+\partial_{z} A^{0}\right] \\
= & \int_{0}^{2 \pi} \frac{d \varphi}{2 \pi} \int_{0}^{c t} d z^{\prime} \int_{0}^{R} \rho d \rho\left\{\frac{\partial_{t}\left(n_{e} \beta_{e}^{z}\right)\left(t_{r}, z^{\prime}\right)}{c \sqrt{\left(z-z^{\prime}\right)^{2}+\rho^{2}}}-\partial_{z} \frac{\widetilde{n_{e 0}}\left(z^{\prime}\right)-n_{e}\left(t_{r}, z^{\prime}\right)}{\sqrt{\left(z-z^{\prime}\right)^{2}+\rho^{2}}}\right\} \\
= & \int_{0}^{c t} d z^{\prime} \int_{0}^{R} d \rho \partial_{\rho}\left\{\frac{\left(z-z^{\prime}\right)\left[n_{e}\left(t_{r}, z^{\prime}\right)-\widetilde{n_{e 0}}\left(z^{\prime}\right)\right]}{\sqrt{\left(z-z^{\prime}\right)^{2}+\rho^{2}}}-\left(n_{e} \beta_{e}^{z}\right)\left(t_{r}, z^{\prime}\right)\right\} \\
= & \int_{0}^{c t} d z^{\prime}\left\{\frac{\left(z-z^{\prime}\right)\left[n_{e}\left(t_{r}, z^{\prime}\right)-\widetilde{n_{e 0}}\left(z^{\prime}\right)\right]}{\sqrt{\left(z-z^{\prime}\right)^{2}+\rho^{2}}}-\left(n_{e} \beta_{e}^{z}\right)\left(t_{r}, z^{\prime}\right)\right\}_{\rho=0}^{\rho=R}
\end{aligned}
$$




$$
\begin{gathered}
=\int_{0}^{c t} d z^{\prime}\left\{\frac{\left(z-z^{\prime}\right)\left\{\left.\left[\widetilde{n_{e 0}}\left(Z_{e}\right) \partial_{y} Z_{e}\right]\left(c t_{r}, y\right)\right|_{y=z^{\prime}}-\widetilde{n_{e 0}}\left(z^{\prime}\right)\right\}}{\sqrt{\left(z-z^{\prime}\right)^{2}+\rho^{2}}}\right. \\
\left.+\frac{1}{c}\left[\widetilde{n_{e 0}}\left(Z_{e}\right) \partial_{t} Z_{e}\right]\left(t_{r}, z^{\prime}\right)\right\}_{\rho=0}^{\rho=R} ;
\end{gathered}
$$

in the derivation we have used: $c t_{r}=c t-\sqrt{\left(z-z^{\prime}\right)^{2}+\rho^{2}}$ for $\boldsymbol{x}=z \hat{\boldsymbol{z}}$; for any differentiable function $f(s)$ the identities $\rho \partial_{z} f\left[t_{r}\right]=\partial_{\rho}\left(z-z^{\prime}\right) f\left(t_{r}\right), \frac{-\rho}{c \sqrt{\left(z-z^{\prime}\right)^{2}+\rho^{2}}} \partial_{t} f\left(t_{r}\right)=\partial_{\rho} f\left(t_{r}\right) ;$ A5 in the last equality. If $\widetilde{n_{e 0}}(Z)=n_{0} \theta(Z)$ we find

$$
\begin{aligned}
& \frac{E^{z}(t, z \hat{\boldsymbol{z}})}{2 \pi e n_{0}}=\sqrt{(z-c t)^{2}+R^{2}}-\sqrt{z^{2}+R^{2}}-|z-c t|+|z| \\
& +\int_{0}^{c t} d z^{\prime}\left\{\left.\frac{\left(z-z^{\prime}\right)\left[\theta\left(Z_{e}\right) \partial_{y} Z_{e}\right]\left(t_{r}, y\right)}{\sqrt{\left(z-z^{\prime}\right)^{2}+\rho^{2}}}\right|_{y=z^{\prime}}+\frac{\theta\left(Z_{e}\right) \partial_{t} Z_{e}}{c}\left(t_{r}, z^{\prime}\right)\right\}_{\rho=0}^{\rho=R}
\end{aligned}
$$

For the suggested FLAME experiment the numerical evaluation by Mathematica in the approximation A1 A4, A9 A10 gives $E^{z}(\bar{t}, \zeta \hat{\boldsymbol{z}}) / 4 \pi e n_{0}=.999$, $E^{z}(t, \zeta \hat{\boldsymbol{z}}) / 4 \pi e n_{0}=1, \quad$ respectively for $\sigma \equiv R / \zeta=1.25$, $\sigma \equiv R / \zeta=1.5$. as claimed after equation (B4).

Proof of (B4). In $\sqrt{\mathrm{B} 5}$ it is $t_{r}=t-\sqrt{R^{2}+\left(z-z^{\prime}\right)^{2}}$ for $\rho=R$; from (B3) and $z^{\prime} \geq 0$ it follows $c t_{r} \leq z^{\prime}$ [25], whence $z_{e}\left(t_{r}, Z\right)=Z, Z_{e}\left(t_{r}, z^{\prime}\right)=z^{\prime}, \partial_{z^{\prime}} Z_{e}\left(t_{r}, z^{\prime}\right)=1$, $\partial_{t} Z_{e}\left(t_{r}, z^{\prime}\right)=0$, so that the integrand in $\mathrm{B} 5$ vanishes for $\rho=R$. For $\rho=0$ it is $t_{r}=t-\left|z^{\prime}-z\right| / c$, and (B5] gives

$$
\begin{aligned}
& \frac{E^{z}(t, z \hat{\boldsymbol{z}})}{2 \pi e}=\int_{0}^{c t} d z^{\prime}\left[s ( z , z ^ { \prime } ) \left\{\left.\left[\widetilde{n_{e 0}}\left(Z_{e}\right) \partial_{y} Z_{e}\right]\left(t-\left|z^{\prime}-z\right| / c, y\right)\right|_{y=z^{\prime}}\right.\right. \\
&\left.\left.-\widetilde{n_{e} 0}\left(z^{\prime}\right)\right\}-\frac{1}{c}\left[\widetilde{n_{e 0}}\left(Z_{e}\right) \partial_{t} Z_{e}\right]\left(t-\left|z^{\prime}-z\right| / c, z^{\prime}\right)\right] \\
&= \int_{0}^{c t} d z^{\prime} s\left(z, z^{\prime}\right)\left\{\widetilde{n_{e 0}}\left(Z_{e}\right) \partial_{z^{\prime}}\left[Z_{e}\left(t-\left|z^{\prime}-z\right| / c, z^{\prime}\right)\right]-\widetilde{n_{e 0}}\left(z^{\prime}\right)\right\} \\
&= \int_{0}^{c t} d z^{\prime} s\left(z, z^{\prime}\right)\left\{\partial_{z^{\prime}} \widetilde{N_{e}}\left[Z_{e}\left(t-\left|z^{\prime}-z\right| / c, z^{\prime}\right)\right]-\widetilde{n_{e 0}}\left(z^{\prime}\right)\right\} \\
&= \int_{z}^{c t} d z^{\prime}\left\{\partial_{z^{\prime}} \widetilde{N_{e}}\left[Z_{e}\left(t+\left(z-z^{\prime}\right) / c, z^{\prime}\right)\right]-n_{e 0}\left(z^{\prime}\right)\right\} \\
& \quad-\int_{0}^{z} d z^{\prime}\left\{\partial_{z^{\prime}} \widetilde{N_{e}}\left[Z_{e}\left(t+\left(z^{\prime}-z\right) / c, z^{\prime}\right)\right]-\widetilde{n_{e 0}}\left(z^{\prime}\right)\right\} \\
&= \widetilde{N_{e}}\left[Z_{e}\left(\frac{z}{c}, c t\right)\right]-\widetilde{N_{e}}(c t)+2\left\{\widetilde{N_{e}}(z)-\widetilde{N_{e}}\left[Z_{e}(t, z)\right]\right\} \\
&+\widetilde{N_{e}}\left[Z_{e}\left(t-\frac{z}{c}, 0\right)\right]=2\left\{\widetilde{N_{e}}(z)-\widetilde{N_{e}}\left[Z_{e}(t, z)\right]\right\}
\end{aligned}
$$

where $s\left(z, z^{\prime}\right)$ stands for the $\operatorname{sign}$ of $z^{\prime}-z$; in the last equality we have used $Z_{e}(z / c, c t)=c t>0, Z_{e}(t, z)>0$, $Z_{e}(t-z / c, 0)<0, \widetilde{N_{e}}\left[Z_{e}(t-z / c, 0)\right]=0$. Note that the condition $c t_{r} \leq z^{\prime}$ is equivalent to requiring that $t$ is less than the sum of the time necessary for the pulse to reach any point $\boldsymbol{x}^{\prime} \in \partial C_{R}$ and of the time lapse necessary for a EM signal to travel from $\boldsymbol{x}^{\prime}$ to $\boldsymbol{x}=z \hat{\boldsymbol{z}}$, what ensures by causality also that at such $t$ the fields in $\boldsymbol{x}$ have not been influenced by the plasma outside $\partial C_{R}$, as anticipated. ii) Upper bound for $\widetilde{E^{\rho}}$ and for the inner displacement of the electrons outside $C_{R}$. As our goal here is to find a sufficient condition for a significant expulsion (expulsion for all electrons initially located in $C_{r}$, $r \gtrsim R / 2$, say), for simplicity of computations we overestimate the $\sigma$ of eq. (8) as follows. For all electrons initially located at points $\overline{\boldsymbol{X}} \simeq R \hat{\boldsymbol{\rho}}+Z \hat{\boldsymbol{z}}$ (with some unit vector $\hat{\boldsymbol{\rho}} \equiv \cos \Phi \hat{\boldsymbol{x}}+\sin \Phi \hat{\boldsymbol{y}}$ ) around the lateral boundary $\partial C_{R}$ of $C_{R}$ we: a) neglect the initial outward boost induced by the pulse on them; b) overestimate the inward component $e \widetilde{E^{\rho}}(t, \boldsymbol{X}) \equiv e E^{\rho}\left[t, \boldsymbol{x}_{e}(\boldsymbol{X})\right]$ of the electric force exerted on them by the charge distribution within $C_{R}$, and consequently their inward displacement $\left|\Delta \rho_{e}\right|$, by the upper bound $E^{\rho}(t, R \hat{\boldsymbol{\rho}})[26]$ c) overestimate $1 / \widetilde{\gamma}_{e}=1 / \sqrt{1+\widetilde{\boldsymbol{u}}_{e}^{2}}$ by $1 / \sqrt{1+{\widetilde{u_{e}^{x}}}^{2}}$ in their relativistic equation of motion. We show that for $t$ in a time lapse growing with $\sigma$

$$
\boldsymbol{E}(t, \boldsymbol{x}) \simeq E^{\rho}(t, \boldsymbol{x}) \hat{\boldsymbol{\rho}}+E^{z}(t, \boldsymbol{x}) \hat{\boldsymbol{z}}, \quad E^{\rho}(t, \boldsymbol{x}) \ll \widetilde{E^{z}}(t, \mathbf{0})
$$

on all points $\boldsymbol{x} \in \partial C_{R}$, i.e. there the electric field is essentially in the $\rho$-direction and much less than the longitudinal one experienced by the $\boldsymbol{X}=\mathbf{0}$ electrons. This is due to geometrical reasons (a look at the coloured charge density plot of fig. 8 center and down may help in getting a qualitative understanding): on one hand, to the delay inherent to the retarded potential itself; on the other, to the fact that the contributions generated by the ions and by the forward boosted electrons sum up on their surface $S_{0}(t)$ of separation, while they partially cancel on $\partial C_{R}$. Therefore tuning $\sigma$ we can make $\left|\Delta \rho_{e}\left(t_{e}, R \hat{\boldsymbol{\rho}}+z \hat{\boldsymbol{z}}\right)\right| \leq R-r$ for all $z$, i.e. the inward displacement of all surface electrons at the estimated time of expulsion $t_{e}=\bar{t}+\Delta t$ small enough not to obstruct the way out to the electrons within $C_{r}$. In particular we find that for the suggested experiments at FLAME it is sufficient to choose $\sigma \equiv R / \zeta=1.25,1.5$ to respectively obtain $r / R \geq 1 / 2,2 / 3$.

For circular polarization $E^{\rho}, \widetilde{E^{\rho}}$ are strictly the same on all the points of this circle $\rho=R, z=0$, in particular on the point $\boldsymbol{x}=R \hat{\boldsymbol{x}}: \quad E^{\rho}(t, R \hat{\boldsymbol{x}})=E^{x}(t, R \hat{\boldsymbol{x}})$. For other (in particular linear) polarizations the relative variation of $E^{\rho}, \widetilde{E^{\rho}}$ along the circle is negligible for modulated periodic oscillations, since the charge distribution has almost cylindrical symmetry due to the fact that transverse displacements almost average to zero in each cycle; therefore also in this case $E^{\rho}(t, R \hat{\boldsymbol{x}}) \simeq E^{x}(t, R \hat{\boldsymbol{x}})$. By $\mathrm{B} 1, \mathrm{~B} 2$

$$
\begin{gathered}
E^{x}(t, x \hat{\boldsymbol{x}})=-\frac{1}{c} \partial_{t} A^{x}-\partial_{x} A^{0} \\
=e \int_{0}^{2 \pi} d \varphi \int_{0}^{c t} d z \int_{0}^{R} \rho d \rho\left\{\frac{\partial_{t}\left(n_{e} \beta_{e}^{x}\right)\left(t_{r}, z\right)}{c \sqrt{z^{2}+C}}-\partial_{x} \frac{n_{0}-n_{e}\left(t_{r}, z\right)}{\sqrt{z^{2}+C}}\right\} \\
=e \int_{0}^{2 \pi} d \varphi \int_{0}^{c t} d z \int_{0}^{R} \rho d \rho\left\{\frac{\partial_{t}\left(n_{e} \beta_{e}^{x}\right)\left(t_{r}, z\right)+c \partial_{x} n_{e}\left(t_{r}, z\right)}{c \sqrt{z^{2}+C}}\right. \\
\left.+\frac{\left[n_{0}-n_{e}\left(t_{r}, z\right)\right](x-\rho \cos \varphi)}{\left|z^{2}+C\right|^{3 / 2}}\right\}
\end{gathered}
$$

where $C=x^{2}+\rho^{2}-2 x \rho \cos \varphi, c t_{r}=c t-\sqrt{z^{2}+C}$ for $\boldsymbol{x}=x \hat{\boldsymbol{x}}$. 


\begin{tabular}{|l|c|c|}
\hline & $R / \zeta=1.25$ & $R / \zeta=1.5$ \\
\hline$\left|\widetilde{\beta_{e}^{\rho}}(\bar{t}, R \hat{\boldsymbol{\rho}})\right|$ & $<0.154$ & $<0.136$ \\
$\left|\widetilde{\Delta \rho_{e}}(\bar{t}, R \hat{\boldsymbol{\rho}})\right| / R$ & $<0.059$ & $<0.04$ \\
average $\tilde{\beta}_{e}^{\rho}$ in $[0, \bar{t}]$ & $\simeq 0.041$ & $\simeq 0.03$ \\
$\left|\widetilde{\beta_{e}^{\rho}}\left(t_{e}, R \hat{\boldsymbol{\rho}}\right)\right|$ & $<0.7$ & $<0.58$ \\
$\left|\widetilde{\Delta \rho_{e}}\left(t_{e}, R \hat{\boldsymbol{\rho}}\right)\right| / R$ & $<0.50$ & $<0.33$ \\
average $\tilde{\beta}_{e}^{\rho}$ in $\left[\bar{t}, t_{e}\right]$ & $<0.43$ & $<0.32$ \\
\hline
\end{tabular}

TABLE II. Main outputs of the inward motion of the lateral electrons in the proposed FLAME experiments

Replacing the approximated $Z_{e}$ A10 in $\mathrm{A} 5$ and the resulting densities in eq. $(\mathrm{B} 9)$, integrating the relativistic equations of motion of the electron $-e \widetilde{E^{x}}=d \widetilde{d p_{e}^{x}} / d t=$ $m c d \widetilde{u_{e}^{x}} / d t, d x_{e} / d t=c \widetilde{\beta_{e}^{x}}=c \widetilde{u_{e}^{x}} / \widetilde{\gamma_{e}}$ with initial conditions $\widetilde{p_{e}^{x}}(0, x \hat{x})=0, \quad x_{e}(0, x \hat{x})=x \hat{x}(x \geq R)$, using overestimates b), c) and Mathematica we numerically find the bounds of table II for $\widetilde{\beta_{e}^{\rho}}(\bar{t}, R \hat{\boldsymbol{\rho}}), \Delta \rho_{e}(t, R \hat{\boldsymbol{\rho}})=\Delta x_{e}(t, R \hat{\boldsymbol{x}})$ in the suggested FLAME experiments.

Finally, we argue that if in 11$)$ we replace $\theta(R-\rho)$ by $e^{-\rho^{2} / R^{2}}$, then outer electrons cause a smaller obstruction to the expulsion of the $\boldsymbol{X} \sim \mathbf{0}$ electrons. In fact, also the outer electrons with initial $\rho \geq R$ get some displacement $\Delta z>0$, therefore (attracted by the ions) cover a smaller inward distance $\Delta \rho_{e}$ in the time necessary for the $\boldsymbol{X} \sim \mathbf{0}$ electrons to come back to $\boldsymbol{x} \sim(0,0, \Delta z)$, and therefore cannot intercept those in a larger cylinder $C_{r}$.
[1] T. Tajima, J.M. Dawson, Phys. Rev. Lett. 43 (1979), 267.

[2] L.M. Gorbunov, and V.I. Kirsanov, Sov. Phys. JETP 66, 290 (1987).

[3] P. Sprangle, E. Esarey, A. Ting, and G. Joyce, Appl. Phys. Lett. 53, 2146 (1988).

[4] A. Irman, M.J.H. Luttikhof, A.G. Khachatryan, F.A. van Goor, J.W.J. Verschuur, H.M.J. Bastiaens, K.-J. Boller, J. Appl. Phys. 102 (2007), 024513.

[5] C. Joshi, Scientific American 294 (2006), 40.

[6] J. Faure, Y. Glinec, A. Pukhov, S. Kiselev, S. Gordienko, E. Lefebvre, J.-P. Rousseau, F. Burgy, V. Malka, Lett. Nat. 431, 541544 (2004).

[7] S. Kalmykov, S. A. Yi, V. Khudik, and G. Shvets, Phys. Rev. Lett. 103 (2009), 135004.

[8] X. Wang, et al., Nature Communications 4 (2013), 1988.

[9] G. Fiore, J. Phys. A: Math. Theor. 47 (2014), 225501.

[10] G. Fiore, Acta Appl. Math. 132 (2014), 261-271.

[11] L.A. Gizzi, et al., Appl. Sci., 3 (2013), 559-580; doi:10.3390/app3030559.

[12] L. Pribyl, L. Juha, G. Korn, et al., in Proc. IBIC2012, Tsukuba, Japan (2012), pp. 482485.

[13] V.I. Eremin, A.V. Korzhimanov, A.V. Kim, Phys. Plasmas 17 (2010), 043102.

[14] J.P. Geindre, R.S. Marjoribanks, P. Audebert, Phys. Rev. Lett. 104 (2010), 135001.

[15] A.A. Gonoskov, A.V. Korzhimanov, A.V. Kim, M. Marklund, A.M. Sergeev, Phys. Rev. E84 (2011), 046403.

[16] $\tau=\tau_{1}+\tau_{2}$, with $\tau_{1}, \tau_{2}$ the time lapses spent during the oscillation respectively in the domain of the elastic and of the constant force; due to the size of the domains it is $\tau_{1} \geq T_{H}^{n r} / 2$. Hence $\tau$ depends on $Z$ and on the oscillation amplitude, but is at the least of the order of $T_{H}^{n r}$.

[17] The longitudinal eq. of motion of the $Z=0$ electrons is

$$
\frac{d \widetilde{p^{z}}}{d t}=m\left[F_{p}^{z}\left(c t-z_{e}\right)-\omega_{p}^{2} z_{e}\right], \quad F_{p}^{z}(\xi) \equiv \frac{(e \lambda)^{2} \eta \partial_{\xi} \epsilon_{s}^{2}(\xi)}{8(m \pi)^{2} c^{3} \gamma(\xi)},
$$

where $\eta=1,1 / 2$ respectively for circular, linear polarization, and $\omega_{p}^{2}=4 \pi e^{2} n_{0} / m$ is the square of the plasma frequency. In the nonrelativistic regime we find $\tilde{\gamma} \simeq 1$, $z_{e}(t) \ll c t, \tilde{p}^{z} \simeq m \dot{z}_{e}$, hence the maximum of $\epsilon_{s}^{2}(\xi)$ and the end of the pulse reach these electrons respectively at $t \simeq \bar{t}=\xi_{0} / c, t \simeq l / c$, and the equation of motion in the bulk reduces to that of a forced harmonic oscillator: $\ddot{z}_{e}=F_{p}^{z}(c t)-\omega_{p}^{2} z_{e}$; the solution with initial conditions $z_{e}(0)=\dot{z}_{e}(0)=0$ is $z_{e}(t)=\int_{0}^{t} d t^{\prime} F_{p}^{z}\left(c t^{\prime}\right) \sin \left[\omega_{p}\left(t-t^{\prime}\right)\right] / \omega_{p}$. Electrons initially located at small $Z>0$ will experience approximately the same longitudinal displacement with a time delay $Z / c . \quad \tilde{F}_{p}^{z} \tilde{v}^{z}=F_{p}^{z} \dot{z}_{e}$ keeps nonnegative if during the pulse $\dot{z}_{e}(t)=\int_{0}^{t} d t^{\prime} F_{p}^{z}\left(c t^{\prime}\right) \cos \left[\omega_{p}\left(t-t^{\prime}\right)\right]$ switches from positive to negative at $t=\bar{t}=\xi_{0} / c$ (only). In particular it must be $z_{e}(t) \geq 0$ for all $t \leq l / c$, and $\xi_{0}, n_{0}, l$ must fulfill $\pi / 4<\xi_{0} \omega_{p} / c<3 \pi / 4$.

If the pulse is so intense to make electrons relativistic the conditions for maximal slingshot loading are more complicated; for an estimate in the proposed experimental conditions see the comments after A7).

[18] In (6) we have chosen the additive constant so that the minima of $U$ w.r.t. $z_{e}$ are equal to zero for all $Z$.

[19] To perform the work integral we have used the relation $\sqrt{z^{2}+R^{2}}=\frac{1}{2} \partial_{z}\left[z \sqrt{z^{2}+R^{2}}+R^{2} \sinh ^{-1} \frac{z}{R}\right]$. We have chosen the additive constant equal to the last three terms so that $U_{R}\left(0^{-}, Z\right)=U\left(0^{+}, Z\right)$.

[20] D. Jovanović, R. Fedele, F. Tanjia, S. De Nicola, L. A. Gizzi, Eur. Phys. J. D66 (2012), 328.

[21] A. Pukhov, Rep. Prog. Phys. 65 (2002), R1-R55.

[22] W. Lu, M. Tzoufras, C. Joshi, F. S. Tsung, J. Vieira, R. A. Fonseca, L. O. Silva, Phys. Rev. ST Accel. Beams 10 (2007), 061301.

[23] As $\partial T_{H} /\left.\partial \zeta\right|_{\zeta=0}=0$, only in the nonrelativistic limit $T_{H} \stackrel{\zeta \rightarrow 0}{\simeq} T_{H}^{n r}=\pi / c \sqrt{K}=\sqrt{\pi m / e^{2} n_{0}}$ is independent of $\zeta$ (nonrelativistic harmonic oscillator).

[24] In fact, B3] implies $t<\left(z^{\prime}+\left|\boldsymbol{x}-\boldsymbol{x}^{\prime}\right|\right) / c$ for all $\boldsymbol{x}^{\prime} \notin C_{R} ; z^{\prime} / c$ is the time when the $R=\infty$ pulse reaches $\boldsymbol{x}^{\prime},\left|\boldsymbol{x}-\boldsymbol{x}^{\prime}\right| / c$ is the time lapse necessary for a EM signal to travel from $\boldsymbol{x}^{\prime}$ to $\boldsymbol{x}$, hence their sum is the time when the information that the charge distribution does not extend indefinitely in the $x y$ plane, but is confined in $C_{R}$, arrives at $\boldsymbol{x}$.

[25] In fact, by the assumptions we find $-2 c t z^{\prime} \leq-2 z z^{\prime}$, (ct$\left.z^{\prime}\right)^{2}=(c t)^{2}-2 c t z^{\prime}+z^{\prime 2} \leq R^{2}+z^{2}-2 z z^{\prime}+z^{\prime 2}=R^{2}+\left(z-z^{\prime}\right)^{2}$ and, taking the square root, $c t_{r} \leq z^{\prime}$.

[26] In fact, $e \tilde{E}^{\rho}$ decreases as $Z$ grows and when the electrons penetrate inside $C_{R}$, as correspondingly the negative contribution due to the electrons displaced beyond $S_{0}(t)$ grows, while the positive one by the ions decreases. 